\title{
MagIK: a Hand Tracking Magnetic Positioning System based on a Kinematic Model of the Hand
}

\author{
Francesco Santoni, Alessio De Angelis, Antonio Moschitta and Paolo Carbone \\ Department of Engineering, University of Perugia, Perugia 06125, Italy \\ Email: \{francesco.santoni, alessio.deangelis, antonio.moschitta, paolo.carbone\}@unipg.it
}

\begin{abstract}
In this paper we present a hand tracking system based on magnetic positioning. A single magnetic node is mounted on each fingertip, and two magnetic nodes on the back side of the hand. A fixed array of receiving coils is used to detect the magnetic field, from which it is possible to infer position and orientation of each magnetic node. A kinematic model of the whole hand has been developed. Starting from the positioning data of each magnetic node, the kinematic model can be used to calculate position and flexion angle of each finger joint, plus the position and orientation of the hand in space. Relying on magnetic fields, the hand tracking system can work also in nonline-of-sight conditions. The gesture reconstruction is validated by comparing it with a commercial hand tracking system based on a depth camera. The system requires a small amount of electronics to be mounted on the hand. This would allow building a light and comfortable data glove that could be used for several purposes: human-machine interface, sign language recognition, diagnostics, and rehabilitation.
\end{abstract}

\section{INTRODUCTION}

C APTURING whole hand gestures has been the subject of intense research for decades [1]-[4]. By capturing hand gestures, it is intended measuring the position and orientation in space of each hand component: fingertips, phalanges, joints, wrist. A hand tracking system is supposed to not only recognize static hand poses but also to track the motion of each part. There are numerous possible applications of hand gesture tracking. Maybe the most known is the implementation of hand tracking as a human-computer interface, particularly in virtual/augmented reality applications [5]-[9]. Another wellknown application, even of considerable commercial interest, is lifelike computer graphics and animation [10]-[12]. As a human-machine interface, hand tracking can be used also to control industrial equipments and robots in real-time [13], [14], even remotely [15]. In combination with machine learning techniques, robots can be taught to perform specific tasks by the repeated observation of human gestures [16]. In a clinical setting, the assessment of the hand kinematics can be exploited for diagnostic and rehabilitation purposes [17]. Hand tracking data and machine learning can be used to recognize characteristic motor symptoms associated with a particular disease (e.g. Parkinson's disease) [18]-[20], or to compare performed gestures with standard references extracted from control sets in targeted rehabilitation exercises [21], [22]. Hand tracking and machine learning are also being studied for automatic sign language recognition [3], [23], [24].

Several techniques have been studied for hand tracking. Vision-based techniques use cameras to capture hand images, then employ machine learning and optimization algorithms to identify features and patterns from which the hand configuration can be inferred [25], [26]. Techniques to identify skin features such as colors, shades, textures have been implemented, as well as contour tracing to discriminate the hand silhouette from the background. These techniques are based on 2D information, and are not particularly efficient. An enhancement solution has been the application on the various hand components of specific markers that can be visually identified [27]. To obtain depth information, stereo cameras, time-of-flight (ToF) or structured light techniques have been used [3], [25]. The LeapMotion controller, that in the following of this paper we will use as a comparison, is a quite well known commercial hand tracking system [20], [28], [29]. It employs three infra-red (IR) LEDs to illuminate the scene, and two IR cameras. Identification and reconstruction of the hand pose are done using stereo-vision techniques. The main draw-back of vision based techniques is that they require lineof-sight conditions, and cannot perform well if, because of the hand orientation or the presence of objects being manipulated, the whole hand is not visible to the cameras [4].

A different solution is the implementation of several sensors mounted on the hand itself, so as not to require an external capture apparatus such as a camera [1]. Stretch or bend sensors have been used to measure joint flexion angles [30], [31], while inertial measurement units (IMU), such as accelerometers or gyroscopes, have been used to obtain informations on position and orientation [5], [17], [32]. Both types of sensors can be integrated in the same system [33]. Generally, the problem with this kind of solution is that, when applied to the whole hand, it may result in a bulky structure that makes movements and objects manipulation uncomfortable.

Another technique that does not require line-of-sight, is based on magnetic field sources and sensors [5], [17], [18]. Magnetic sources can be mounted on the hand, while sensors are external [34], or, conversely, the magnetic field source is external, while magnetic sensors are mounted on the hand, as, for example, using the commercial magnetic positioning system Polhemus [35], [36]. In a different approach, both sources and sensors are mounted on the hand [37], [38], but in this case only the relative distance and orientation can be measured, hence the hand configuration can be reconstructed, but not its absolute position and orientation in space.

A further distinction in hand tracking approaches is between discriminative and generative methods [2], [4]. In the first case, hand gesture reconstruction is mainly based on collected 
data, e.g. position measurements from the IMUs mounted on each moving part of the hand or pattern recognition and classification in visual-based methods. In the second case, a priori parametric and kinetimatic models of the hand are used to predict the gesture. This distinction is not rigorous, since of course sensing and visual data are at any rate needed to complete and correct the model. Many approaches are then a hybrid of discriminative and generative techniques.

In this work, we present a hand tracking technique based on an already existent magnetic positioning system (MPS) [39]. The MPS can track the position and orientation of six independent magnetic nodes. In order to track the hand, we used all the nodes: two nodes fixed on the back side of the hand, and one node fixed on each fingertip (with six nodes only, it was thus not possible to track the pinkie, but in principle, the technique can be easily extended). We used a hybrid approach by developing a kinematic model of the hand. Once positions and orientations of the nodes are known from the MPS, it is possible to use the kinematic model to obtain position and flexion of each joint, and the position and orientation of the whole hand in space, i.e. with respect to the fixed reference frame of the MPS. We called our system MagIK (Magnetic and Inverse Kinematics). We tested MagIK by comparing the gesture reconstruction with that obtained by the LeapMotion system.

Relying on magnetic fields, the system can work in non-lineof-sight conditions. Moreover, since only six small magnetic nodes and a small supply unit have to be mounted on the hand, this would allow building a light and comfortable data glove that could be used for all the purposes listed above: humanmachine interface, sign language recognition, diagnostic and rehabilitation, and so on.

\section{DESCRIPTION OF THE SYSTEM AND METHODS}

\section{A. The magnetic positioning system}

A complete description of the MPS can be found in [39]; here we give just a short summary. Magnetic active nodes are small solenoids coupled with a capacitor (resonant LC circuits), and supplied with an alternating voltage, thus generating an oscillating magnetic field. Also receivers are solenoids coupled with capacitors. The varying magnetic field of the active nodes induces a measurable voltage on receivers. By measuring the induced voltage on each receiver, and inverting a mathematical model [40], it is possible to infer the position and the orientation of each active node, where the orientation is defined as the direction of the solenoid symmetry axis. Receivers are mounted on a stable wooden structure, shown in Figure 1 with its reference frame. There are 24 receivers inserted into carved slots. 16 sensors are mounted under the horizontal plane, arranged on a regular $4 \times 4$ grid, the distance between sensors being $8 \mathrm{~cm}$; 4 sensors are mounted at the center of each one of the vertical planes, positioned on the vertices of an $8 \mathrm{~cm}$ side square. The active volume of the system is a $30 \times 30 \times 30 \mathrm{~cm}^{3}$ box.

Voltages on each receiver are acquired by analogto-digital converters (ADC). Texas Instruments Delfino TMS320F28379D microcontroller units (MCU) have been

\section{a)}

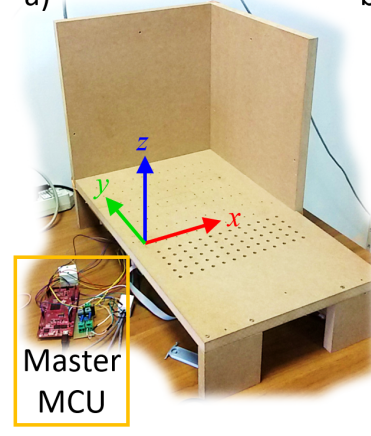

b)
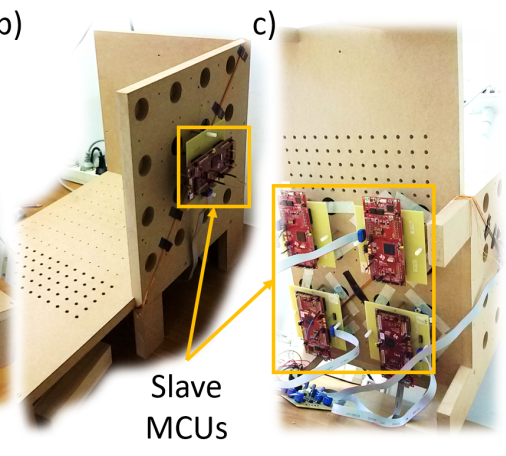

Fig. 1. A picture of the magnetic positioning system. a) Front view. The reference frame is shown. The grid carved on the $x y$ plane is used for the calibration procedure described in [41]. Receivers are not visible from the front. b) Side view of the $y z$ plane; receivers are inserted into round carved slots; only four receivers are mounted at the center of each vertical plane; signals on the receivers are acquired by microcontroller units equipped with four ADCs each. c) Bottom view. Sixteen receivers are mounted under the $x y$ plane with four microcontrollers.

used to build the system. Each MCU is equipped with four ADCs; six MCUs are thus needed to acquire data from all the receivers. A further MCU is used in a master-slave configuration to control all the others, simultaneously triggering the acquisition on each slave MCU, and collecting all the data. The master $\mathrm{MCU}$ is connected to a personal computer via serial port. A custom software reads all the voltages and a timestamp in microseconds from the master, and then calculates position and orientation of each node, i.e. three cartesian coordinates, and three direction cosines. The MPS error on position $\epsilon_{r}$ is defined as the Euclidean distance between measured and real positions. By choosing a reference set of positions, an empirical distribution for $\epsilon_{r}$ is obtained, as reported in [39]. Taking the average and the standard deviation of this distribution, the Euclidean error of the MPS can be estimated as $\hat{\epsilon}_{r}=3.5 \pm 1.9 \mathrm{~mm}$. Similarly, the MPS angular error $\epsilon_{\theta}$ is defined as the angle between measured and real orientations, and its estimate is $\hat{\epsilon}_{\theta}=2.6^{\circ} \pm 1.5^{\circ}$. The MPS can perform 62 measurements per second almost constantly, since computation times are variable. That measurement rate was obtained on a computer equipped with a six-core Intel i7-8750H CPU at $2.20 \mathrm{GHz}$, running Windows 10 , and it is high enough to measure also fast hand movements; indeed the MPS was already used to measure the hand tremor associated with Parkinson's disease [42], by mounting the active nodes on the hand.

Magnetic nodes have been mounted on the hand as shown in Figure 2. Nodes 1 and 2 are mounted on the hand's back. Their relative distance is fixed as $5 \mathrm{~cm}$, and their orientation is the same. Node 2 is positioned with its center over the metacarpophalangeal joint of the middle finger (MCP-III, see Figure 3 for bone names, numbers and abbreviations). The support of nodes 1 and 2 is placed parallel to the third metacarpal bone. Nodes 3-6 are fixed at the center of distal phalanges by means of small plastic rings. A magnified view of a single magnetic node is provided in the inset of Figure 2. By referring to the reference frame indicated in the same picture, only rotations around $v$ and $w$ axes can be measured with the 


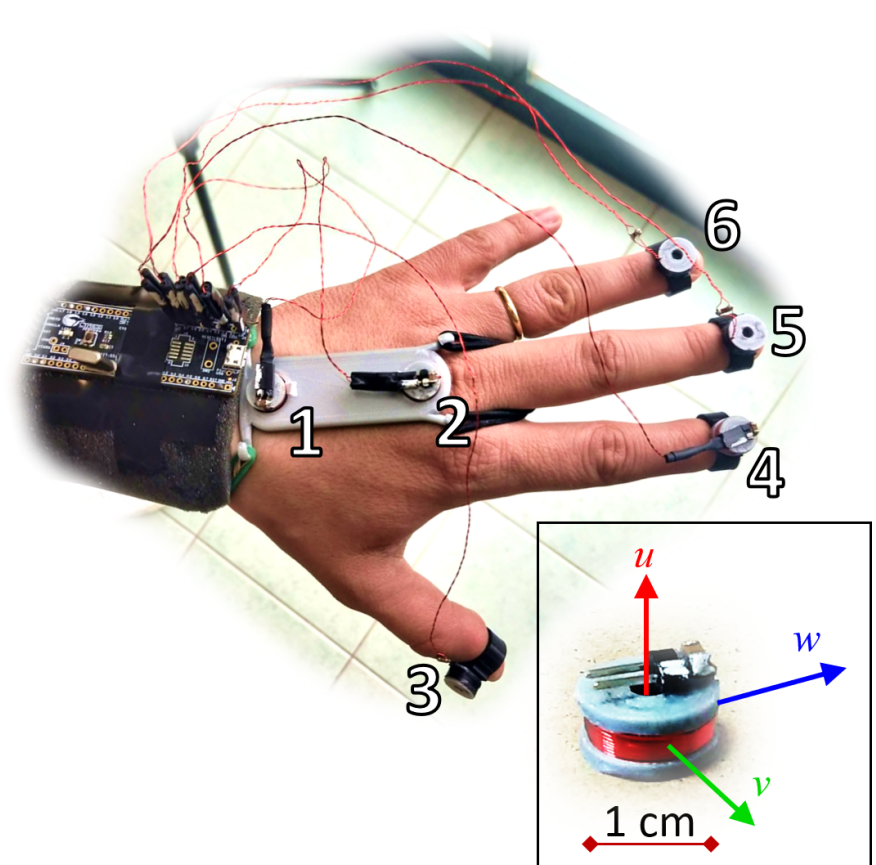

Fig. 2. Magnetic nodes mounted on the hand. The supply unit is tied to the wrist. Two magnetic nodes are mounted on the hand's back; they are used to measure the position and orientation of the hand in space. One node is mounted on each fingertip (the pinkie excepted) in order to measure finger position and flexion with respect to metacarpus. A single magnetic node is shown in the inset; rotations around its $u$ axis cannot be measured by the MPS because of the cylindrical symmetry of the node.

MPS; rotations around the $u$ axis cannot be detected because of the cylindrical symmetry of the node (and of its magnetic field accordingly). The position and the orientation of each node are thus specified by five degrees of freedom (5-DOF): three cartesian coordinates and two angles; the MPS provides three direction cosines for the orientation, but actually they are not independent, only two components - e.g. elevation and azimuth - being sufficient to identify the orientation of a node in the reference frame of the MPS. In order to completely identify the position and the orientation of the hand, a 6-DOF measurement is needed. For this reason a single node fixed on the hand's back was not sufficient. By using two 5-DOF nodes, the symmetry around the $u$ axis of Figure 2 is broken, with the direction of the line through the centers of nodes 1 and 2 providing the remaining DOF. The orientation of the hand is thus identified by the orientation of the third metacarpal bone. The hand reference frame is shown in Figure 3(b); the origin $O$ is identified with the magnetic node 1 .

Magnetic nodes are power-supplied using square waves generated with a Cypress PSoC 5LP microcontroller tied on the wrist [39]. Each node is supplied by using a different frequency. To discriminate different nodes by Fourier analysis, Frequency Division Multiple Access (FDMA) is used [43]. Operating frequencies are in the range 176-186 kHz. Any external electromagnetic interference within this range might impair the accuracy of the measurement system, while frequencies outside the same range are effectively cut out by the resonant receiving coils [44]. a)
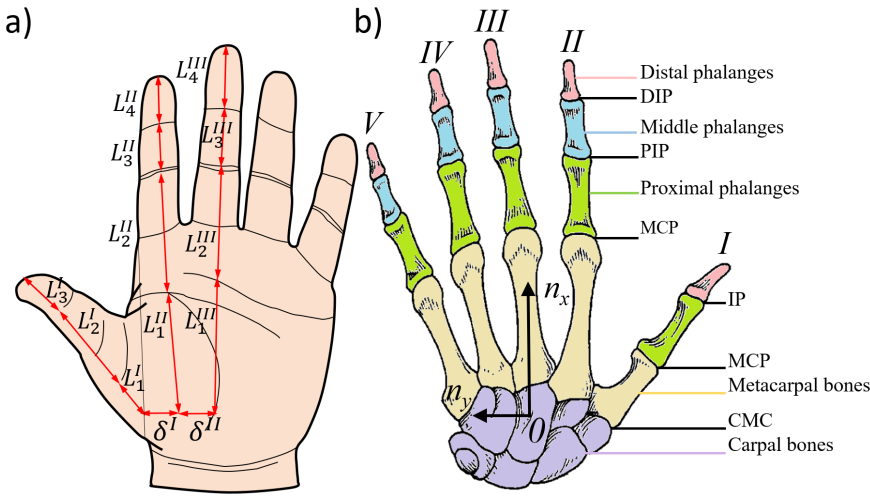

Fig. 3. a) Palm view. The lengths of the various hand segments have been defined by using skin lines as reference, instead of actual bone lengths. Lengths for fingers IV and V are not shown, since they are defined analogously. b) Back view. Hand parts: CMC - carpometacarpal joints; MCP metacarpophalangeal joints; PIP - proximal interphalangeal joints; DIP - distal interphalangeal joints; IP - interphalangeal joint.

\section{B. Kinematic model of the hand}

Kinematic models of the human hand have been studied for decades, mainly with the aim to design robotic hands [45][49]. We used the model described in [47] [48], with some modification.

1) Forward kinematics: The kinematics of the hand is described by using the modified Denavit-Hartenberg (MDH) parameters introduced by Craig [50]. Hand segments are shown in Figure 3. Instead of using actual bone lengths, we considered skin creases as a more realistic reference to define lengths $L_{j}^{i}$; indeed the effective hand gesture, as well as the effective interaction with objects, are defined by the external surface of the hand.

All abduction and flexion angles are illustrated in Figure 4. CMC abduction angles $\vartheta_{\mathrm{CMC}, \mathrm{a}}^{i}$ for fingers $i=\mathrm{II}-\mathrm{V}$ are fixed; $\vartheta_{\mathrm{CMC}, \mathrm{a}}^{i}$ is the angle between the finger axis and the base of the hand; $\vartheta_{\mathrm{CMC}, \mathrm{a}}^{\mathrm{III}}=\pi / 2$ by definition, while the other fixed angles will be determined during the calibration phase illustrated in Section III-B. Fixed abduction angles $\vartheta_{\mathrm{CMC}, \mathrm{a}}^{i}$ are not defined in the model of [47], [48], but they are required to obtain the convergence of fingers II-V when they are clenched while keeping DIP joints extended, a characteristic feature of the human hand [51], [52]. MCP mobile abduction angles for fingers II-V are measured by $\theta_{\mathrm{MCP}, \mathrm{a}}^{i}$. Flexion angles are defined in Figure 4(c); $\theta_{\mathrm{CMC}, \mathrm{f}}^{\mathrm{III}}=0$ by definition, since CMC flexion is measured with respect to the direction of the third metacarpal bone, chosen as indicator of the global orientation of the hand; $\theta_{\mathrm{MCP}, \mathrm{f}}^{\mathrm{I}}$ and $\theta_{\mathrm{IP}, \mathrm{f}}^{\mathrm{I}}$ for the thumb are not shown, since they are analogous to $\theta_{\mathrm{MCP}, \mathrm{f}}^{i}$ and $\theta_{\mathrm{DIP}, \mathrm{f}}^{i}$. The $\mathrm{CMC}$ abduction angle for the thumb is measured by $\theta_{\mathrm{CMC}, \mathrm{a}}^{\mathrm{I}}$, as shown in Figure 4(b). The torsion of the thumb around its axis is measured by the twist angle $\alpha_{\mathrm{MCP}}^{\mathrm{I}}$, as shown in Figure 4(a); thumb twist was not used in [47], [48], but it is essential for a realistic model [51], otherwise the proper opposition of the thumb, with the pulp touching the palm, would be mechanically impossible. Thumb twist is not a fixed angle. The torsion of the thumb changes as it is flexed towards the palm; a quantitative espression cannot be found in the literature, but 
a)

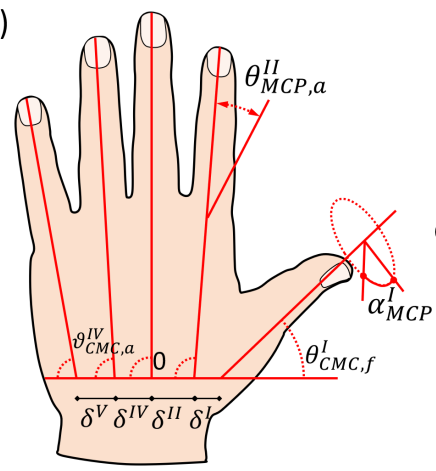

b)

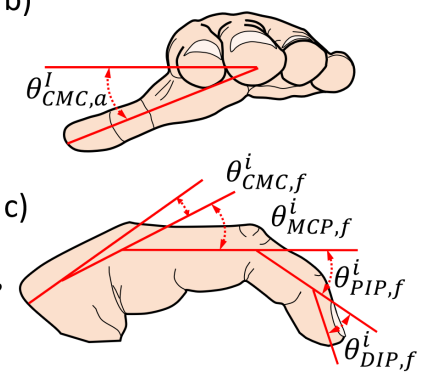

Fig. 4. Angles defined in the kinematic model of the hand. a) Fixed abduction angles $\vartheta_{\mathrm{CMC} \text {, a }}^{i}$ and mobile abduction angles $\theta_{\mathrm{MCP}, \mathrm{a}}^{i}$ for fingers II-V (same greek letter, but different symbols have been used to distinguish fixed and mobile angles). Angles are shown only for some of the fingers, the others being analogous. $\alpha_{\mathrm{MCP}}^{\mathrm{I}}$ is the twist angle of the thumb. The flexion angle of the thumb at the CMC joint is indicated with $\theta_{\mathrm{CMC}, \mathrm{f}}^{\mathrm{I}}$. The distance between finger bases is indicated with $\delta^{i}$. b) Thumb CMC abduction angle $\theta_{\mathrm{CMC}, \mathrm{I}}^{\mathrm{I}}$ seen from fingertips. c) Flexion angles for fingers $i=\mathrm{II}-\mathrm{V}$. For the thumb, $\theta_{\mathrm{MCP}, \mathrm{f}}^{\mathrm{I}}$ and $\theta_{\mathrm{IP}, \mathrm{f}}^{\mathrm{I}}$ are analogous to $\theta_{\mathrm{MCP}, \mathrm{f}}^{i}$ and $\theta_{\mathrm{DIP}, \mathrm{f}}^{i}$, hence they are not shown. $\theta_{\mathrm{CMC}, \mathrm{f}}^{i}$ is measured with respect to the direction of the third metacarpal bone, chosen as indicator of the global orientation of the hand; hence $\theta_{\mathrm{CMC}, \mathrm{f}}^{\mathrm{III}}=0$ always by definition.
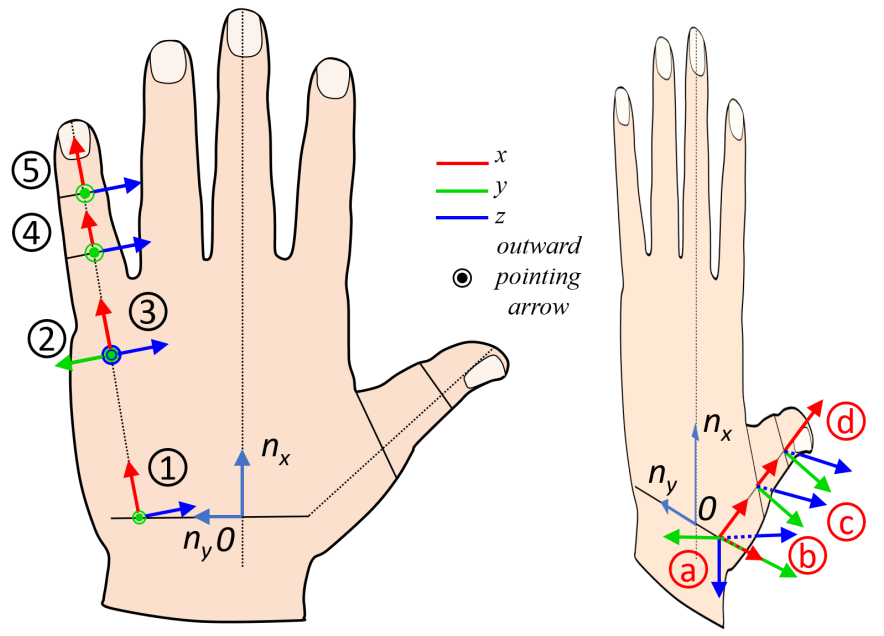

Fig. 5. Local reference frames attached to each hand link. The link chain has the same structure for fingers II-V, hence it is conveniently shown only for the pinkie. According with the MDH convention, $x$ axes are always oriented along link directions, while $z$ axes are attached to the rotation axis of each link ( $y$ axes are thus fixed by the right hand rule). For fingers II-V, links 1 , 3,4 and 5 are flexion links, while 2 is an abduction link. For finger I, links $\mathrm{b}, \mathrm{c}$ and $\mathrm{d}$ are flexion links, while $\mathrm{a}$ is an abduction link.

we obtained good results by using:

$$
\alpha_{\mathrm{MCP}}^{\mathrm{I}} \equiv \alpha\left(\theta_{\mathrm{CMC}, \mathrm{f}}^{\mathrm{I}}\right)=\frac{\alpha_{0}}{2}\left(2-\frac{\theta_{\mathrm{CMC}, \mathrm{f}}^{\mathrm{I}}-\theta_{\mathrm{CMC}, \mathrm{f}, \min }^{\mathrm{I}}}{\theta_{\mathrm{CMC}, \mathrm{f}, \max }^{\mathrm{I} a}-\theta_{\mathrm{CMC}, \mathrm{f}, \min }^{\mathrm{min}}}\right),
$$

where $\alpha_{0}$ is the torsion angle measured when the thumb is extended.

Initially, we used exactly the same model illustrated in [47], [48], but we found that the reconstruction of the gesture was not accurate, hence we introduced the modifications illustrated above. Thus, there are 5 DOFs for the thumb, counting the twist angle, and 5 DOFs for each one of the other fingers; but since $\theta_{\mathrm{CMC}, \mathrm{f}}^{\mathrm{III}}$ is fixed, there are a total of 24 DOFs, i.e. 24 mobile angles, represented by the MDH parameters $\theta$ 's, plus the parameter $\alpha_{\mathrm{MCP}}^{\mathrm{I}}$ for thumb twist.

TABLE I

MDH PARAMETERS FOR FINGERS II-V.

\begin{tabular}{|c|c|c|c|c|c|}
\hline & Joint & $a$ & $\mathrm{~d}$ & $\theta$ & $\alpha$ \\
\hline 5 & $\mathrm{DIP}$ & $L_{3}^{i}$ & 0 & $\theta_{\mathrm{DIP}, \mathrm{f}}^{i}$ & 0 \\
\hline 4 & $\mathrm{PIP}$ & $L_{2}^{i}$ & 0 & $\theta_{\mathrm{PIP}, \mathrm{f}}^{i}$ & 0 \\
\hline 3 & $\mathrm{MCP}, \mathrm{f}$ & 0 & 0 & $\theta_{\mathrm{MCP}, \mathrm{f}}^{i}$ & $\pi / 2$ \\
\hline 2 & $\mathrm{MCP}, \mathrm{a}$ & $L_{1}^{i}$ & 0 & $\theta_{\mathrm{MCP}, \mathrm{a}}^{i}$ & $-\pi / 2$ \\
\hline 1 & $\mathrm{CMC}, \mathrm{f}$ & 0 & 0 & $\theta_{\mathrm{CMC}, \mathrm{f}}^{i}$ & $\pi / 2$ \\
\hline
\end{tabular}

TABLE II

MDH PARAMETERS FOR THE THUMB.

\begin{tabular}{|c|c|c|c|c|c|}
\hline & Joint & $a$ & $\mathrm{~d}$ & $\theta$ & $\alpha$ \\
\hline $\mathrm{d}$ & $\mathrm{IP}$ & $L_{2}^{\mathrm{I}}$ & 0 & $\theta_{\mathrm{IP}, \mathrm{f}}^{\mathrm{I}}$ & 0 \\
\hline $\mathrm{c}$ & $\mathrm{MCP}$ & $L_{1}^{\mathrm{I}}$ & 0 & $\theta_{\mathrm{MCP}, \mathrm{f}}^{\mathrm{I}}$ & $-\alpha_{\mathrm{MCP}}^{\mathrm{I}}$ \\
\hline $\mathrm{b}$ & $\mathrm{CMC}, \mathrm{f}$ & 0 & 0 & $\theta_{\mathrm{MCP}, \mathrm{f}}^{\mathrm{I}}$ & 0 \\
\hline $\mathrm{a}$ & $\mathrm{CMC}, \mathrm{a}$ & $\delta^{\mathrm{I}}$ & 0 & $\theta_{\mathrm{CMC}, \mathrm{a}}^{\mathrm{I}}$ & $\pi / 2$ \\
\hline
\end{tabular}

According with the MDH convention, a reference frame is attached to each hand link, as shown in Figure 5; $x$ axes are always oriented along link directions, while $z$ axes are attached to the rotation axis of each link ( $y$ axes are thus fixed by the right hand rule). Mobile $\theta$ angles measure rotations around $z$ axes; $\alpha$ angles measure link twists around $x$ axes (only the thumb has a torsion); link lengths are measured along $x$ axes, and are represented by the MDH parameter $a$; the last MDH parameter is $d$, a measure of link shifts along $z$ axes, but, for the kinetic model of the hand, $d$ is always zero. The complete set of MDH parameters is reported in Tables I and II.

Rotations and translations can be more easily treated by using a compact formalism based on homogeneous coordinates; i.e., in this section, when we use a position vector, it is intended to be a vector of the form $\mathbf{r}=[x, y, z, 1]^{T}$, unless otherwise stated. Rotations and translations are represented by using $4 \times 4 \mathrm{MDH}$ matrices. For the finger $i$, we define $T_{k}^{i}\left(\theta_{k}^{i}, \alpha_{k}^{i}, a_{k}^{i}\right)$ as the matrix transforming the coordinates with respect to the reference frame $k$, to coordinates with respect to the frame $k-1$ :

$$
T_{k}^{i}=\left(\begin{array}{cccc}
\cos \theta_{k}^{i} & \sin \theta_{k}^{i} & 0 & a_{k}^{i} \\
-\sin \theta_{k}^{i} \cos \alpha_{k}^{i} & \cos \theta_{k}^{i} \cos \alpha_{k}^{i} & -\sin \alpha_{k}^{i} & -d \sin \alpha \\
-\sin \theta_{k}^{i} \sin \alpha_{k}^{i} & \cos \theta_{k}^{i} \sin \alpha_{k}^{i} & \cos \alpha_{k}^{i} & d \cos \alpha \\
0 & 0 & 0 & 1
\end{array}\right)
$$

The MDH matrix represents the sequence of operations: translation $d$ along $z$ axis $\rightarrow$ rotation $\theta$ around $z$ axis $\rightarrow$ translation $a$ along $x$ axis $\rightarrow$ twist $\alpha$ around $x$ axis. Since $d$ is always zero, it is omitted from now on. The $\sin \theta_{k}^{i}$ terms should have opposite signs according with the MDH convention, and the angles of Figure 4(b) and 4(c) would have negative values; since it is more natural, for the hand model, to use positive values for those angles, we changed the signs of their respective sines. Let us now consider fingers II-V. For 
finger $i$ we thus have:

$$
\begin{aligned}
T_{5}^{i} & \equiv T_{5}^{i}\left(\theta_{\mathrm{DIP}, \mathrm{f}}^{i}, 0, L_{3}^{i}\right), \\
T_{4}^{i} & \equiv T_{4}^{i}\left(\theta_{\mathrm{PIP}, \mathrm{f}}^{i}, 0, L_{2}^{i}\right), \\
T_{3}^{i} & \equiv T_{3}^{i}\left(\theta_{\mathrm{MCP}, \mathrm{f}}^{i}, \pi / 2,0\right), \\
T_{2}^{i} & \equiv T_{2}^{i}\left(\theta_{\mathrm{MCP}, \mathrm{a}}^{i},-\pi / 2, L_{1}^{i}\right), \\
T_{1}^{i} & \equiv T_{1}^{i}\left(\theta_{\mathrm{CMC}, \mathrm{f}}^{i}, \pi / 2,0\right), \\
T_{0}^{i} & \equiv T_{0}^{i}(-\pi / 2,0,0) T_{0}^{i}\left(\vartheta_{\mathrm{CMC}, \mathrm{a}}^{i}, 0, \pm \delta^{i}\right),
\end{aligned}
$$

where $T_{0}^{i}$, a product of two constant MDH matrices, has been introduced in order to transform coordinates from the finger base reference frame, to the hand reference frame $O$. We can then write the complete $\mathrm{MDH}$ matrix $T^{i}$ as

$$
T^{i}\left(\boldsymbol{\theta}^{i}, \boldsymbol{\alpha}^{i}, \boldsymbol{a}^{i}\right)=\prod_{k=0}^{5} T_{k}^{i} .
$$

A similar treatment holds for the thumb:

$$
\begin{aligned}
T_{d}^{\mathrm{I}} & \equiv T_{d}^{\mathrm{I}}\left(\theta_{\mathrm{IP}, \mathrm{f}}^{\mathrm{I}}, 0, L_{2}^{\mathrm{I}}\right), \\
T_{c}^{\mathrm{I}} & \equiv T_{c}^{\mathrm{I}}\left(\theta_{\mathrm{MCP}, \mathrm{f}}^{\mathrm{I}}, \alpha_{\mathrm{MCP}}^{\mathrm{I}}, L_{1}^{\mathrm{I}}\right), \\
T_{b}^{\mathrm{I}} & \equiv T_{b}^{\mathrm{I}}\left(\theta_{\mathrm{CMC}, \mathrm{f}}^{\mathrm{I}}, 0,0\right), \\
T_{a}^{\mathrm{I}} & \equiv T_{a}^{\mathrm{I}}\left(\theta_{\mathrm{CMC}, \mathrm{a}}^{\mathrm{I}} \pi / 2, \delta^{\mathrm{I}}\right), \\
T_{0}^{\mathrm{I}} & \equiv T_{0}^{\mathrm{I}}(\pi / 2,0,0) .
\end{aligned}
$$

The complete MDH matrix for the thumb can be computed as in (4).

The position $\boldsymbol{R}^{\mathrm{I}}$ of the magnetic node 3, with respect to frame (d), is fixed, as well as the position of magnetic nodes 4-6 $\boldsymbol{R}^{i}$, with respect to frame (5): $\boldsymbol{R}^{\mathrm{I}}=\left[L_{3}^{\mathrm{I}} / 2,0,0,1\right]^{T}$ and $\boldsymbol{R}^{i}=\left[L_{4}^{i} / 2,0,0,1\right]^{T}$. The position $\boldsymbol{r}_{0}^{i}$ of all the nodes, with respect to the hand frame $O$, of the magnetic nodes 3-6 can thus be obtained as:

$$
\boldsymbol{r}_{0}^{i}=T^{i}\left(\boldsymbol{\theta}^{i}, \boldsymbol{\alpha}^{i}, \boldsymbol{a}^{i}\right) \boldsymbol{R}^{i}
$$

Let also $T_{\text {rot }}^{i}$ be the $3 \times 3$ rotation submatrix of $T^{i}$, i.e. the matrix obtained from $T^{i}$ by removing both the fourth row and the fourth column. Unit vectors representing the orientation of nodes 3-6, with respect to frames (5) and (d), have the same expression: $\hat{\mathbf{N}}^{i}=[0,1,0]^{T}$. The orientations of nodes $3-6$, with respect to frame $O$, are thus given by:

$$
\hat{\boldsymbol{n}}_{0}^{i}=T_{\mathrm{rot}}^{i}\left(\boldsymbol{\theta}^{i}, \boldsymbol{\alpha}^{i}\right) \hat{\mathbf{N}}^{i} .
$$

2) Inverse kinematics: Positions and orientations of all the magnetic nodes, with respect to the fixed reference frame of Figure 1, are measured by means of the MPS. In Section II-B4, it will be illustrated how the reference frame $O$, attached to the hand, is determined from the position and the orientation of nodes 1 and 2, in the MPS reference frame. Let $\boldsymbol{r}$ be the position of the origin $O$, and $\hat{\boldsymbol{n}}_{x}, \hat{\boldsymbol{n}}_{y}, \hat{\boldsymbol{n}}_{z}$ the axes of the hand frame with respect to the MPS. Thus, $\boldsymbol{r}_{0}^{i}$ and $\hat{\boldsymbol{n}}_{0}^{i}$ are related to positions and orientations measured in the MPS frame by an invertible matrix, as:

$$
\begin{aligned}
\mathbf{r}_{\mathrm{MPS}}^{i} & =\left(\begin{array}{cccc}
\hat{\mathbf{n}}_{x} & \hat{\mathbf{n}}_{y} & \hat{\mathbf{n}}_{z} & \mathbf{r} \\
0 & 0 & 0 & 1
\end{array}\right) \mathbf{r}_{0}^{i}, \\
\hat{\mathbf{n}}_{\mathrm{MPS}}^{i} & =\left(\begin{array}{ccc}
\hat{\mathbf{n}}_{x} & \hat{\mathbf{n}}_{y} & \hat{\mathbf{n}}_{z}
\end{array}\right) \hat{\mathbf{n}}_{0}^{i},
\end{aligned}
$$

where homogeneous coordinates are not used to express $\boldsymbol{r}$. Since the matrices in (8) represent rotations and a translation, i.e. operations that can be reversed by definition, those matrices are clearly invertible. Once $\boldsymbol{r}_{0}^{i}$ and $\hat{\boldsymbol{n}}_{0}^{i}$ have been obtained, the reconstruction of the hand gesture becomes the problem of finding the set of values $\left\{\boldsymbol{\theta}^{i}, \alpha_{\mathrm{MCP}}^{\mathrm{I}}\right\}$ for which (6) and (7) hold; this is the inverse kinematics problem.

In [47], [48], an analytic solution for the inverse kinematics is provided. There are however a number of problems to directly apply the same approach to our model. Analytical formulas in [47], [48] are quite long and difficult to handle, and in our model they would get even more complicated, since for the thumb we have added the $\alpha_{\mathrm{MCP}}^{\mathrm{I}} \mathrm{DOF}$; moreover, there are several denominators that can approach zero, compromising the numerical stability of the solution; lastly, in order to apply the analytic solution, a 6-DOF measurement of the distal phalanx is needed, but by using magnetic nodes, we get only a 5-DOF measurement, as explained in Section II-A.

We used instead a numerical approach to the inverse kinematics problem, by minimizing the squared modulus of the five-components vector obtained by combining (6) and (7) as:

$$
\begin{gathered}
L S^{i}=\left\|\left[10\left(\boldsymbol{r}_{0}^{i}-T^{i} \boldsymbol{R}^{i}\right), 1-\hat{\boldsymbol{n}}_{0}^{i} \cdot T_{\text {rot }}^{i} \hat{\boldsymbol{N}}^{i}\right]^{T}\right\|^{2}, \\
\hat{\boldsymbol{\theta}}^{i}=\underset{\theta}{\arg \min L S^{i}}\left(\boldsymbol{\theta}^{i}\right) .
\end{gathered}
$$

A separate optimization problem has thus to be solved for each finger. The scale factor 10 on the position components (first four components) has been introduced in order to ensure that all the five components have the same order of magnitude, assuming that lengths are measured in meters. The scale factor is important to lower the error on the final solution.

3) Initialization of the algorithm: Several local minima are possible, hence the optimization algorithm has to be properly conditioned, in order to converge toward the correct solution. The first strategy is to initialize the algorithm with values $\hat{\boldsymbol{\theta}}^{i}$ as close as possible to the true ones. This is easily done by keeping the hand naturally open, as in Figure 3, at the very beginning of the tracking; in this way all angles are approximately zero. After the first hand pose has been reconstructed, at each subsequent measurement, $\hat{\boldsymbol{\theta}}^{i}$ is initialized with the values of the previous pose. We verified that a similar approach is needed also with the LeapMotion system, indeed its reconstruction of the hand pose is often wrong, if the first recognition is not performed while keeping the hand open.

The initialization strategy is still not sufficient for a good convergence, the DOF that was missing when discussing the analytical solution, is still missing also when using the numerical approach. Also the positioning error of the MPS affects the convergence. The solution is to introduce some constraints, as it will be explained in Section III-A. With proper initialization and constraints, a stable convergence to correct minima is attained.

Magnetic nodes are tracked in real-time by the MPS; all the data are stored, and then processed in order to reconstruct hand poses. We did not develop a stand-alone software to track the hand in real-time. In order to solve the optimization 


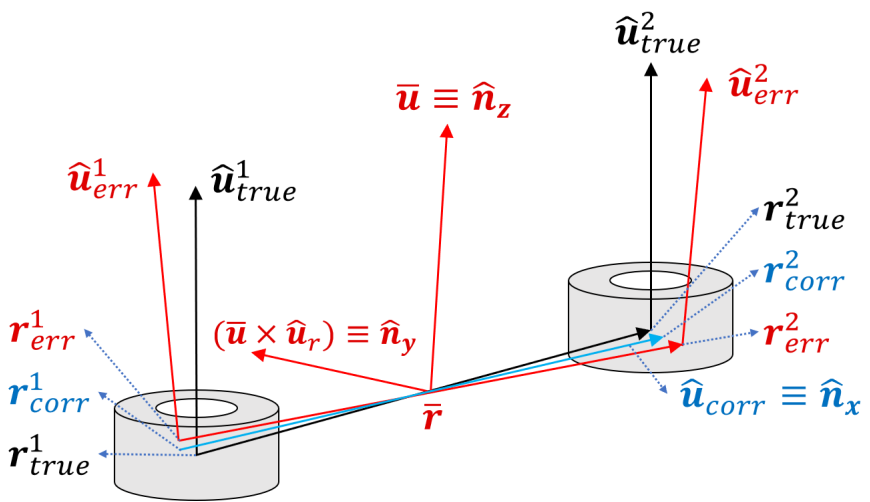

Fig. 6. Illustration of the geometrical method used to determine the hand reference frame from the position and orientation of nodes 1 and 2, measured by means of the MPS. $\boldsymbol{r}^{1}$ and $\boldsymbol{r}^{2}$ are the positions of nodes 1 and 2 respectively, while $\hat{\boldsymbol{u}}^{1}$ and $\hat{\boldsymbol{u}}^{2}$ are their orientations.

problem, we used the Matlab function lsqnonlin, a nonlinear least-square data-fitting algorithm using a trust-region method, that also takes lower and upper bounds $\boldsymbol{\theta}_{\min }^{i}$ and $\boldsymbol{\theta}_{\max }^{i}$ as arguments. By running the optimization algorithm on a computer equipped with a six-core Intel $17-8750 \mathrm{H} \mathrm{CPU}$ at $2.20 \mathrm{GHz}$, running Windows 10 , it has been possible to obtain $\approx 55$ hand reconstructions per second, a measurement rate that is high enough also for potential real-time applications; this rate could also be increased by implementing the algorithm in a compiled language, such as $\mathrm{C} / \mathrm{C}++$, or by adopting parallelization strategies, as, for example, reconstructing all the fingers simultaneously by running five concurrent threads.

4) Determination of the hand reference frame: At each measurement, in order to reconstruct the hand reference frame of Figure 3(b), the orientation of node 1 or 2 can be used to define the $z$ axis, while the direction of the line passing through the measured positions of node 1 and 2 can be used to define the $x$ axis; the $y$ axis would then be determined accordingly by using the right-hand rule. The accuracy of this reconstruction is improved by applying a geometrical method as follows. A 5-DOF measurement of a single node is not sufficient to determine the reference frame, but since nodes 1 and 2 are kept at fixed distance $L$ and at the same orientation, they provide redundant information, allowing error reduction.

The geometrical construction is illustrated in Figure 6. True values of position and orientation $\left(\boldsymbol{r}_{\text {true }}^{1}, \boldsymbol{r}_{\text {true }}^{2}, \hat{\boldsymbol{u}}_{\text {true }}^{1}, \hat{\boldsymbol{u}}_{\text {true }}^{2}\right)$ are not known; their corresponding error-affected values $\left(\boldsymbol{r}_{\text {err }}^{1}, \boldsymbol{r}_{\text {err }}^{2}, \hat{\boldsymbol{u}}_{\mathrm{err}}^{1}, \hat{\boldsymbol{u}}_{\mathrm{err}}^{2}\right)$ are measured by means of the MPS. The middle point and the average orientation are obtained as:

$$
\overline{\boldsymbol{r}}=\frac{1}{2}\left(\boldsymbol{r}_{\mathrm{err}}^{1}+\boldsymbol{r}_{\mathrm{err}}^{2}\right), \quad \overline{\boldsymbol{u}}=\frac{\hat{\boldsymbol{u}}_{\mathrm{err}}^{1}+\hat{\boldsymbol{u}}_{\mathrm{err}}^{2}}{\left\|\hat{\boldsymbol{u}}_{\mathrm{err}}^{1}+\hat{\boldsymbol{u}}_{\mathrm{err}}^{2}\right\|} .
$$

This approach relies on the fact that independent errors are summed in quadrature, hence if $\epsilon_{r}$ is the positioning error of the MPS, the error affecting $\overline{\boldsymbol{r}}$ can be estimated as $\sqrt{2} \epsilon_{r} / 2<$ $\epsilon_{r}$; a similar result holds for the angular error. The $z$ direction $\hat{\boldsymbol{n}}_{z}$ is identified with $\overline{\boldsymbol{u}}$, while the $y$ direction is obtained from the direction $\hat{\boldsymbol{u}}_{r}$ through $\boldsymbol{r}_{\mathrm{err}}^{1}$ and $\boldsymbol{r}_{\mathrm{err}}^{2}$ as:

$$
\hat{\boldsymbol{u}}_{r}=\frac{\boldsymbol{r}_{\mathrm{err}}^{2}-\boldsymbol{r}_{\mathrm{err}}^{1}}{\left\|\boldsymbol{r}_{\mathrm{err}}^{2}-\boldsymbol{r}_{\mathrm{err}}^{1}\right\|}, \quad \hat{\boldsymbol{n}}_{y}=\overline{\boldsymbol{u}} \times \hat{\boldsymbol{u}}_{r} .
$$
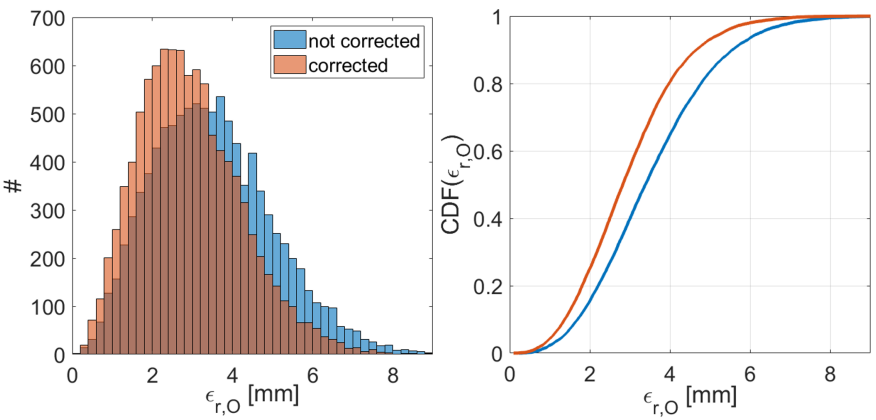

Fig. 7. Simulated distributions of $\epsilon_{r, O}$ for the position of the origin $O$ of the hand frame, and cumulative distribution functions, before and after the geometrical correction has been applied.
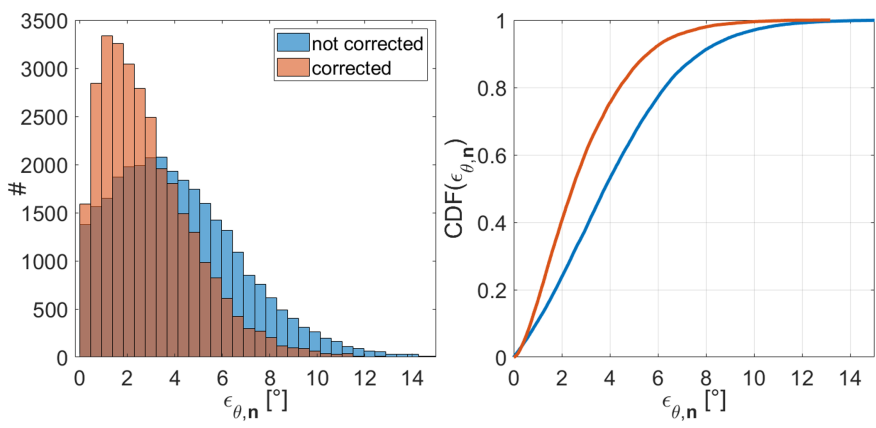

Fig. 8. Simulated distributions of $\epsilon_{\theta, \boldsymbol{n}}$ for the orientation of hand frame axes, and cumulative distribution functions, before and after the geometrical correction has been applied.

Then, in order to ensure $\hat{\boldsymbol{u}}_{r}$ being perpendicular also to $\hat{\boldsymbol{n}}_{z}, \hat{\boldsymbol{u}}_{r}$ is corrected by rotating it around $\hat{\boldsymbol{n}}_{y}$ through $\overline{\boldsymbol{r}}$, thus obtaining $\hat{\boldsymbol{u}}_{\text {corr }}$ (blue line in Figure 6), that can be taken as $\hat{\boldsymbol{n}}_{x}$. Finally, nodes positions are recalculated as:

$$
\boldsymbol{r}_{\text {corr }}^{1}=\overline{\boldsymbol{r}}-\frac{L}{2} \hat{\boldsymbol{u}}_{\text {corr }}, \quad \boldsymbol{r}_{\text {corr }}^{2}=\overline{\boldsymbol{r}}+\frac{L}{2} \hat{\boldsymbol{u}}_{\text {corr }} .
$$

The position of the origin $O$ of the hand reference frame is identified with the corrected vector $\boldsymbol{r}_{\text {corr }}^{1}$.

In order to estimate the reduction of the Euclidean error $\epsilon_{r, O}$ on the position of the origin $O$, and the reduction of the angular error $\epsilon_{\theta, n}$ on the orientation of frame axes, due to the geometrical correction, we performed a simple Monte Carlo (MC) simulation. We generated $10^{4}$ random samples of $\boldsymbol{r}_{\text {true }}^{1}, \boldsymbol{r}_{\text {true }}^{2}, \hat{\boldsymbol{u}}_{\text {true }}^{1}, \hat{\boldsymbol{u}}_{\text {true }}^{2}$ within the active volume of the MPS, and then assuming a Gaussian error on position and orientation, thus obtaining a sample set for $\boldsymbol{r}_{\mathrm{err}}^{1}, \boldsymbol{r}_{\mathrm{err}}^{2}, \hat{\boldsymbol{u}}_{\mathrm{err}}^{1}, \hat{\boldsymbol{u}}_{\mathrm{err}}^{2}$. The Gaussian error has been chosen as to reproduce the average accuracy of the MPS on a single node as reported in [39]. For nodes position, a zero mean random translation, with standard deviation $\sigma_{x y z}=2.2 \mathrm{~mm}$, has been added along each dimension. For nodes orientation, a zero mean random rotation, with standard deviation $\sigma_{\phi}=4.2^{\circ}$, has been applied around random axes. The resulting distributions of $\epsilon_{r, O}$ and $\epsilon_{\theta, \boldsymbol{n}}$ are shown in Figure 7 and 8. The average error is $\bar{\epsilon}_{r, O}=3.5 \mathrm{~mm}$, and $\bar{\epsilon}_{\theta, \boldsymbol{n}}=4.1^{\circ}$. We then applied the geometrical correction to this set, obtaining the corrected distributions of Figure 7 and 8 . The corrected average error is $\bar{\epsilon}_{r, O \text {,corr }}=2.9 \mathrm{~mm}$, and $\bar{\epsilon}_{\theta, \boldsymbol{n} \text {,corr }}=2.9^{\circ}$. The corrected distributions of $\epsilon_{r, O \text {,corr }}$ and $\epsilon_{\theta, \boldsymbol{n} \text {,corr }}$ can be equivalently 
TABLE III

STATIC CONSTRAINTS

\begin{tabular}{|c|c|c|c|c|c|}
\hline & Thumb & Index & Middle & Ring & Pinkie \\
\hline$\theta_{\mathrm{CMC}, \mathrm{a}}$ & {$\left[0^{\circ}, 90^{\circ}\right]$} & Not Defined & N.D. & N.D. & N.D. \\
\hline$\theta_{\mathrm{CMC}, \mathrm{f}}$ & {$\left[0^{\circ}, 90^{\circ}\right]$} & {$\left[0^{\circ}, 5^{\circ}\right]$} & 0 & {$\left[0^{\circ}, 10^{\circ}\right]$} & {$\left[0^{\circ}, 15^{\circ}\right]$} \\
\hline$\theta_{\mathrm{MCP}, \mathrm{a}}$ & N.D. & {$\left[-30^{\circ}, 30^{\circ}\right]$} & {$\left[-22^{\circ}, 22^{\circ}\right]$} & {$\left[-22^{\circ}, 22^{\circ}\right]$} & {$\left[-25^{\circ}, 25^{\circ}\right]$} \\
\hline$\theta_{\mathrm{MCP}, \mathrm{f}}$ & {$\left[0^{\circ}, 80^{\circ}\right]$} & {$\left[-40^{\circ}, 90^{\circ}\right]$} & {$\left[-40^{\circ}, 90^{\circ}\right]$} & {$\left[-40^{\circ}, 90^{\circ}\right]$} & {$\left[-40^{\circ}, 90^{\circ}\right]$} \\
\hline$\theta_{\mathrm{PIP}, \mathrm{f}}$ & N.D. & {$\left[0^{\circ}, 110^{\circ}\right]$} & {$\left[0^{\circ}, 110^{\circ}\right]$} & {$\left[0^{\circ}, 120^{\circ}\right]$} & {$\left[0^{\circ}, 135^{\circ}\right]$} \\
\hline$\theta_{\mathrm{DIP}, \mathrm{f}}$ & {$\left[-10^{\circ}, 80^{\circ}\right]$} & {$\left[-5^{\circ}, 80^{\circ}\right]$} & {$\left[-5^{\circ}, 80^{\circ}\right]$} & {$\left[-5^{\circ}, 80^{\circ}\right]$} & {$\left[-5^{\circ}, 90^{\circ}\right]$} \\
\hline
\end{tabular}

obtained by adding a corrected Gaussian error with standard deviations $\sigma_{x y z \text {,corr }}=1.8 \mathrm{~mm}$ and $\sigma_{\phi, \text { corr }}=1.7^{\circ}$.

\section{HAND MODEL CONSTRAINTS AND CALIBRATION}

The introduction of some constraint is essential to attain a stable convergence of the optimization algorithm, as explained in Section II-B2. The human hand has natural constraints that can be easily implemented. Moreover, all fixed parameters of Table I and II $-L_{j}^{i}, \delta^{i}, \vartheta_{\mathrm{CMC}, \mathrm{a}}^{i}, \alpha_{0}^{\mathrm{I}}-$ have to be determined. Instead of measuring directly each parameter, we developed a fast calibration procedure making use of the same MPS.

\section{A. Constraints}

Hand constraints can be divided into static and dynamic constraints. Static constraints are defined by the range of motion of each joint, i.e. the minimum and maximum values of each mobile angle. Dynamic constraints are relations between different joints, expressed as equations.

Static constraints are reported in Table III as given in [47]. Static constraints can be directly passed as arguments to the Isqnonlin algorithm, as stated in Section II-B2. As reported in the table, $\theta_{\mathrm{CMC} \text {, } \mathrm{II}}^{\mathrm{III}}$ ixed to zero; however, the optimization algorithm becomes unstable if $\theta_{\max }=\theta_{\min }=0$ are set. A simple workaround is to set $\theta_{\max }=-\theta_{\min }=0.1^{\circ}$; by using this setting, the algorithm is stable, while the error introduced on the angle is negligible.

As it will be shown in Section IV-A, by using the previous constraints, stable convergence and high accuracy is attained, as long as the measurement error on the position and the orientations of magnetic nodes is low; this proves the validity of the model. But, given the actual accuracy of the MPS, in general the result is not a natural pose of the hand. Indeed, because of the physiology of the human hand, it is almost impossible, or at least difficult, for most people to flex the DIP joint, without flexing the PIP joint too. Capturing this feature is important in order to reconstruct a natural hand pose, given the measurement error of the MPS. The following dynamic constraint has thus been used [47], [48]:

$$
\theta_{\mathrm{DIP}, \mathrm{f}}^{i} \approx 0.67 \theta_{\mathrm{PIP}, \mathrm{f}}^{i},
$$

where $i=\mathrm{II}-\mathrm{V}$. A different ratio (0.88) is also reported in literature [53]. The chosen value is the most commonly used, and we obtain good results by using it. Relation (14) is only approximately valid, and there can be substantial variability among different people [53]. Such constraint can be applied by appending to the vector in (9) the following component:

$$
w\left(\theta_{\mathrm{DIP}, \mathrm{f}}^{i}-0.67 \theta_{\mathrm{PIP}, \mathrm{f}}^{i}\right) .
$$

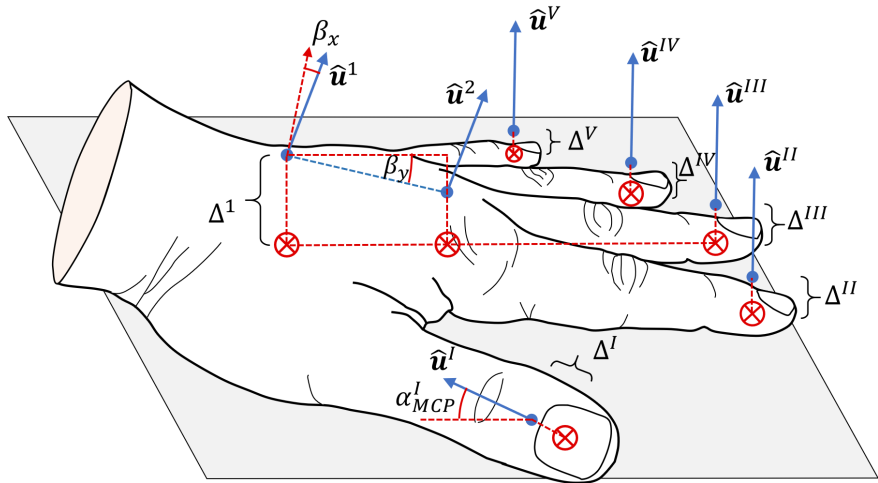

Fig. 9. A representation of the calibration method to align the hand frame to the palm plane.

In general the least-square solution does not match the exact ratio of the angles according with constraint (14), but by raising the weight $w$, it is possible to force the constraint towards its exact ratio, while with lower values of $w$, the computed values of the ratio are spread around the exact value. In order to get a natural pose, $w=1$ is a good choice. The constraint can be turned off by setting $w=0$; this could be useful when fingers do not move freely, but objects and obstacles are present, invalidating (14). When the hand is grasping objects of various shapes, different sets of suitable constraints are available in the literature [47], [48]; they could be implemented in the same fashion, and switched on and off by using a weight factor.

Another physiological constraint is the limitation of the MCP abduction angle as fingers are flexed [52]; for $\theta_{\mathrm{MCP}, \mathrm{f}}>$ 0 it can be expressed as:

$$
\begin{aligned}
\theta_{\mathrm{MCP}, \mathrm{a}, \mathrm{dmax}} & =\left(1-\frac{\theta_{\mathrm{MCP}, \mathrm{f}}}{\theta_{\mathrm{MCP}, \mathrm{f}, \mathrm{smax}}}\right) \theta_{\mathrm{MCP}, \mathrm{a}, \mathrm{smax}}, \\
\theta_{\mathrm{MCP}, \mathrm{a}, \mathrm{dmin}} & =-\theta_{\mathrm{MCP}, \mathrm{a}, \mathrm{dmax}} .
\end{aligned}
$$

smax indicates static maxima as reported in Table III; dmax and dmin are the dynamical maxima and minima: for each measurement, they are calculated by using the $\theta_{\mathrm{MCP}, \mathrm{f}}$ value obtained at the previous pose reconstruction, then are passed as upper and lower boundaries to the lsqnonlin algorithm.

\section{B. Model calibration}

For the calibration phase, the hand is steadily laid on a horizontal support within the active volume of the MPS, as in Figure 9. The hand pose has to be natural and relaxed: fingers II-V are extended with zero abduction; the thumb is open without stretching. The position and the orientation of all nodes is measured by means of the MPS; it is convenient to keep the hand steady for a few seconds, as to perform many measurements on the same position: we used 500 repeated measurements to calibrate the model. In Figure 9, the unit vectors $\hat{\boldsymbol{u}}^{i}$ represents the orientation of all nodes, while their application point is the actual node position on the hand, as in Figure 2. In the kinematic model presented in Section II-B, it is assumed that all the nodes lay on a plane parallel to the palm, and intersecting the central axis of each finger; let us call them virtual nodes, represented by red circles in Figure 9. 


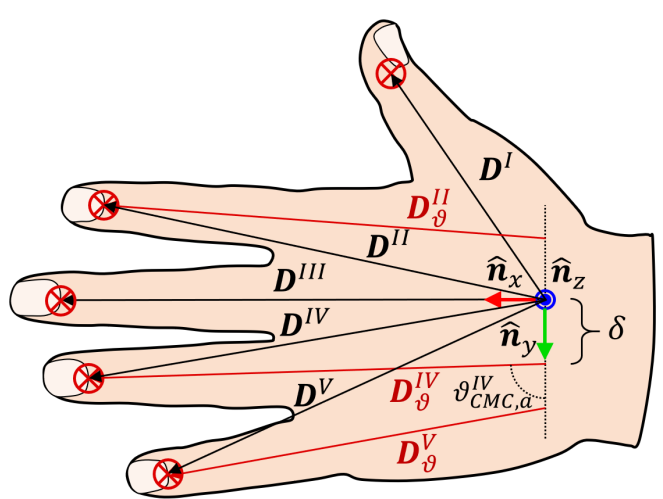

Fig. 10. Distances $D^{i}$ between virtual nodes measured by means of the MPS, and used to calibrate the model.

Virtual node orientations are perpendicular to the palm plane. The first step of the calibration method is to align all the nodes with their virtual counterparts. The second step is to determine all the other parameters: $L_{j}^{i}, \delta^{i}, \vartheta_{\mathrm{CMC}, \mathrm{a}}^{i}, \alpha_{\mathrm{CMC}}^{\mathrm{I}}$. The whole procedure has been implemented in a Matlab script.

1) Aligning the nodes to the palm plane: As shown in Figure 9 , there are fixed biases $\left(\Delta^{i}, \beta_{x}, \beta_{y}\right)$ between real and virtual nodes. Once these biases have been determined, they are constantly applied to each hand measurement, before calculating the pose through inverse kinematics. The orientation of the hand reference frame with respect to the MPS, obtained by the geometrical method of Section II-B4, gives the angles $\beta_{x}$ and $\beta_{y}$, respectively a rotation around the axis connecting node 1 and 2, and around the $y$ axis of the hand frame. The shifts $\Delta^{i}$ are measured along directions $\hat{\boldsymbol{u}}^{i}$ (for node 1 after the corrections for $\beta_{x}$ and $\beta_{y}$ have been applied). A single $\Delta^{\text {ref }}$ value for a reference node must be known a priori, then all the other nodes are shifted along $\hat{\boldsymbol{u}}^{i}$, as to have the same $z$ coordinate as the reference virtual node, thus fixing all $\Delta^{i}$ values.

2) Determination of model parameters: The twist parameter $\alpha_{\mathrm{MCP}}^{\mathrm{I}}$ for the thumb is obtained by directly measuring, by means of the MPS, the elevation angle of $\hat{\boldsymbol{u}}^{\mathrm{I}}$.

For the next steps, it is assumed that the unbiased hand reference frame has already been obtained. The distances $\boldsymbol{D}^{i}$ between the virtual nodes on fingertips and virtual node 1, as shown in Figure 10, are extracted from MPS data. By means of average values of anthropometric hand data from an extended survey [54], we determined a set of proportions between distances $D^{i}$ and the lengths of finger segments $L_{j}^{i}$ and $\delta^{i}$, as defined in Figure 3(a). The distance between finger bases $\delta$ is assumed to be the same for all fingers. A good approximation for $\delta$ is:

$$
\delta=0.09 D^{\mathrm{III}} .
$$

In general we define $D^{i}=\left\|\boldsymbol{D}^{i}\right\|$. Fixed angles $\vartheta_{\mathrm{CMC}, \mathrm{a}}^{i}$ can be calculated by using simple trigonometry. Let us define $\boldsymbol{D}_{\vartheta}^{i}$ as in Figure 10, i.e.:

$$
\begin{aligned}
\boldsymbol{D}_{\vartheta}^{\mathrm{II}} & =\mathbf{D}^{\mathrm{II}}+\delta \hat{\mathbf{n}}_{y}, \\
\boldsymbol{D}_{\vartheta}^{\mathrm{IV}} & =\mathbf{D}^{\mathrm{IV}}-\delta \hat{\mathbf{n}}_{y}, \\
\boldsymbol{D}_{\vartheta}^{\mathrm{V}} & =\mathbf{D}^{\mathrm{V}}-2 \delta \hat{\mathbf{n}}_{y} ;
\end{aligned}
$$

TABLE IV

PROPORTIONALITY COEFFICIENTS FOR FINGER SEGMENT LENGTHS

\begin{tabular}{|l|l|r|r|r|r|}
\hline $\mathbf{c}$ & $\mathbf{i}=\mathbf{I}$ & II & III & IV & $\mathbf{V}$ \\
\hline $\mathbf{j}=\mathbf{1}$ & 0.258 & 0.305 & 0.343 & 0.316 & 0.328 \\
\hline $\mathbf{2}$ & 0.332 & 0.423 & 0.377 & 0.393 & 0.383 \\
\hline $\mathbf{3}$ & 0.324 & 0.167 & 0.183 & 0.181 & 0.163 \\
\hline $\mathbf{4}$ & N.D & 0.210 & 0.195 & 0.220 & 0.251 \\
\hline
\end{tabular}

while $\boldsymbol{D}_{\vartheta}^{\mathrm{I}} \equiv \boldsymbol{D}^{\mathrm{I}}$ and $\boldsymbol{D}_{\vartheta}^{\mathrm{III}} \equiv \boldsymbol{D}^{\mathrm{III}}$. Fixed angles are thus obtained as:

$$
\vartheta_{\mathrm{CMC}, \mathrm{a}}^{i}=\cos ^{-1}\left(\frac{\mathbf{D}_{\vartheta}^{i}}{D_{\vartheta}^{i}} \cdot \hat{\mathbf{n}}_{y}\right),
$$

where $i=\mathrm{II}, \mathrm{IV}, \mathrm{V}$, and recall that $\vartheta_{\mathrm{CMC}, \mathrm{a}}^{\mathrm{III}}=\pi / 2$ by definition. Finally, segment lengths $L_{j}^{i}$ are obtained as:

$$
L_{j}^{i}=c_{j}^{i} D_{\vartheta}^{i}
$$

where the coefficients $c_{j}^{i}$ are reported in Table IV.

\section{HAND TRACKING TEST}

In order to test the hand tracking system, first, we have investigated the accuracy of the kinematic model by means of a MC simulation; then, we evaluated the hand pose reconstruction by tracking a real hand, and by confronting the results with those of the LeapMotion system on a series of benchmarks.

\section{A. Testing the model}

We generated a set of configurations for fingers I and IV by sweeping $\theta$ values over their whole ranges, as reported in Table III. By (6) and (7), positions $\boldsymbol{r}_{0, \text { true }}$ and orientations $\hat{\boldsymbol{n}}_{0, \text { true }}$ of the magnetic nodes mounted on distal phalanges have been calculated. The standard deviation of the zero mean Gaussian error on nodes position and orientation, in the hand reference frame, can be estimated as $\sigma_{x y z \text {,hand }}=\sqrt{\sigma_{x y z}^{2}+\sigma_{x y z \text {,corr }}^{2}}$ and $\sigma_{\phi, \text { hand }}=\sqrt{\sigma_{\phi}^{2}+\sigma_{\phi, \text { corr }}^{2}}$, where $\sigma_{x y z}, \sigma_{\phi}, \sigma_{x y z \text {,corr }}$ and $\sigma_{\phi, \text { corr }}$ are defined as in Section II-B4. By adding the Gaussian error, a set of $\boldsymbol{r}_{0, \text { err }}$ and $\hat{\boldsymbol{n}}_{0, \text { err }}$ values is obtained.

In order to check the validity of the optimization algorithm, $\boldsymbol{r}_{0, \text { true }}$ and $\hat{\boldsymbol{n}}_{0, \text { true }}$ without Gaussian error were first substituted into (9), as to verify that the reconstructed $\theta$ values
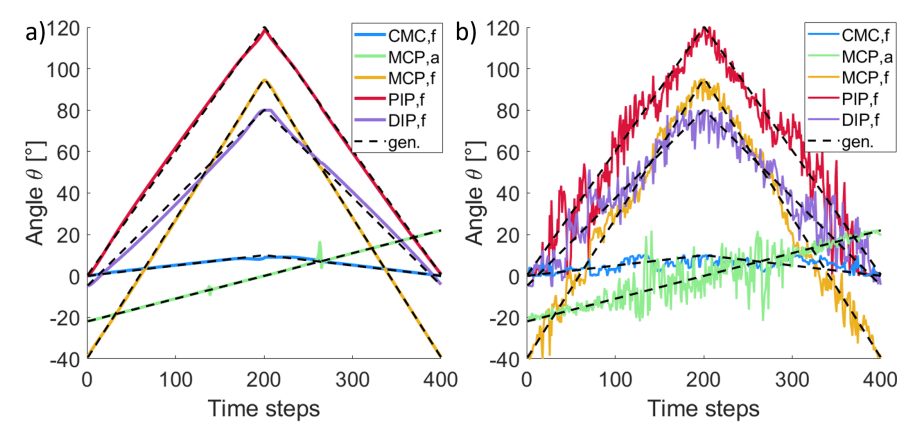

Fig. 11. Generated and reconstructed $\theta$ values for the ring finger. a) Reconstruction from exact $\boldsymbol{r}_{0, \text { true }}$ and $\hat{\boldsymbol{n}}_{0, \text { true }}$ values (no Gaussian error added. b) Reconstruction from Gaussian error affected $\boldsymbol{r}_{0, \text { err }}$ and $\hat{\boldsymbol{n}}_{0, \text { err }}$ values. 

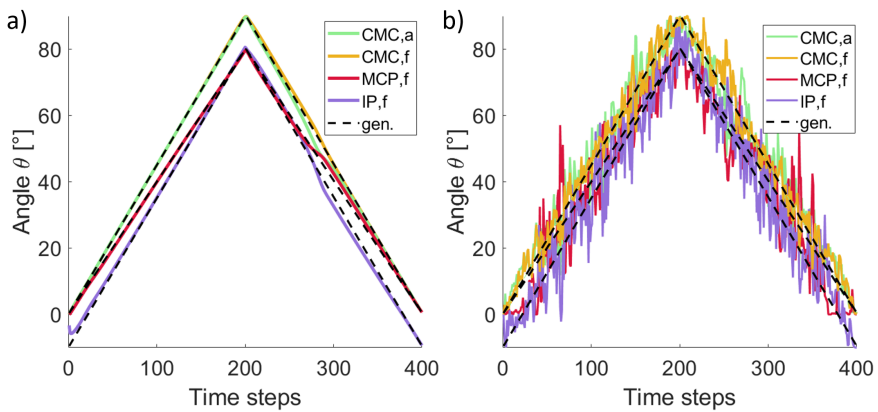

Fig. 12. Generated and reconstructed $\theta$ values for the thumb. a) Reconstruc-

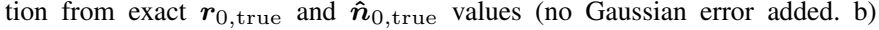
Reconstruction from Gaussian error affected $\boldsymbol{r}_{0, \text { err }}$ and $\hat{\boldsymbol{n}}_{0, \text { err }}$ values.

would match the generated ones. Resulting $\theta$ values are shown in Figure 11(a) and Figure 12(a) for the ring finger and the thumb respectively. The accordance is quite good, considering that we did not use strict criteria for the convergence of the optimization algorithm (we set both step tolerance and function tolerance to $10^{-3}$ ); the CDFs of the Euclidean error on the reconstructed position of the fingertip, and of the angular error on the reconstructed orientation of the distal phalanx are reported in Figure 13 and 14, respectively for the ring finger and the thumb. In both cases, the mean position error and the mean angular error are less than $1 \mathrm{~mm}$ and $2^{\circ}$ respectively. The accuracy of the result could be easily improved by adopting stricter convergence criteria, but this would impact significantly on the computing time; with a view to future real-time applications, we kept the computing time low by choosing less stringent tolerance values.

Since in real life situations, position and orientation of all the nodes are always known with limited accuracy, it is fundamental to check the inverse kinematics by substituting $\boldsymbol{r}_{0, \text { err }}$ and $\hat{\boldsymbol{n}}_{0, \text { err }}$ into (9). In Figure 11(b) and Figure 12(b), reconstructed $\theta$ values are reported for the ring finger and the thumb respectively. Results look quite noisy, but the corresponding Euclidean error on the position of the fingertip, and the angular error on the orientation of the distal phalanx (Figure 13 and 14) stay contained within acceptable ranges for a realistic reconstruction of the hand pose, as it will be shown in the following. For the ring finger, the mean position and angular errors are $\bar{\epsilon}_{r \text {,hand }}^{\mathrm{IV}}=4.7 \mathrm{~mm}$ and $\bar{\epsilon}_{\theta \text {,hand }}^{\mathrm{IV}}=5.5^{\circ}$; for the thumb, $\bar{\epsilon}_{r \text {,hand }}^{\mathrm{I}}=5.2 \mathrm{~mm}$ and $\bar{\epsilon}_{\theta, \text { hand }}^{\mathrm{I}}=5.7^{\circ}$.

We estimated position and angular errors for each joint and phalanx; in Figure 15, it is reported the CDF of the Euclidean error on the position, in the hand frame, of the joints and the tip of the ring finger, and the angular error on the orientation of the phalanges; analogous results for the thumb are reported in Figure 16. We checked also the reconstruction accuracy by adding only a fraction of the Gaussian error $\left(\frac{1}{2}, \frac{1}{4}\right.$ and $\left.\frac{1}{8}\right)$; results are reported in Figure 13 and 14; as it was expected, as the Gaussian error on $\boldsymbol{r}_{0, \text { err }}$ and $\hat{\boldsymbol{n}}_{0, \mathrm{err}}$ is reduced, the accuracy of the reconstruction approaches the numerical accuracy of the optimization algorithm, with tolerances being set as above. This is a clear evidence that the only factor limiting the accuracy of the pose reconstruction, is the measurement noise of the MPS; by using a more accurate
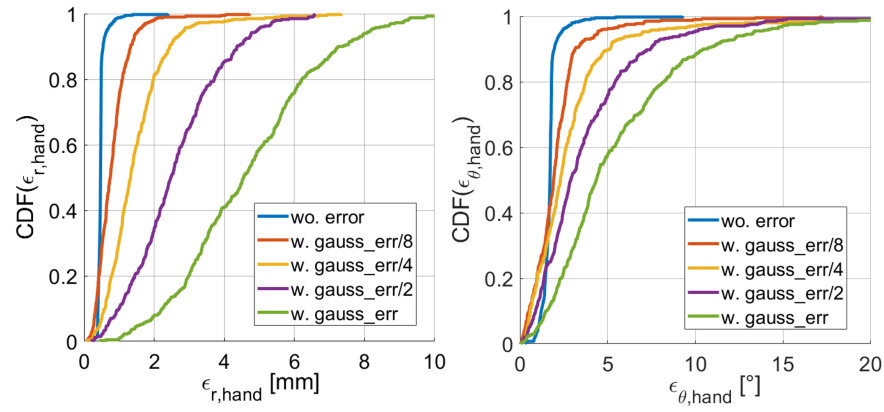

Fig. 13. Euclidean error $\epsilon_{r \text {, hand }}$ on the reconstructed position of the ring fingertip with respect to hand reference frame, and angular error $\epsilon_{\theta \text {, hand }}$ on the orientation of the ring distal phalanx. Results are shown for several Gaussian error values, and for the control set without error.
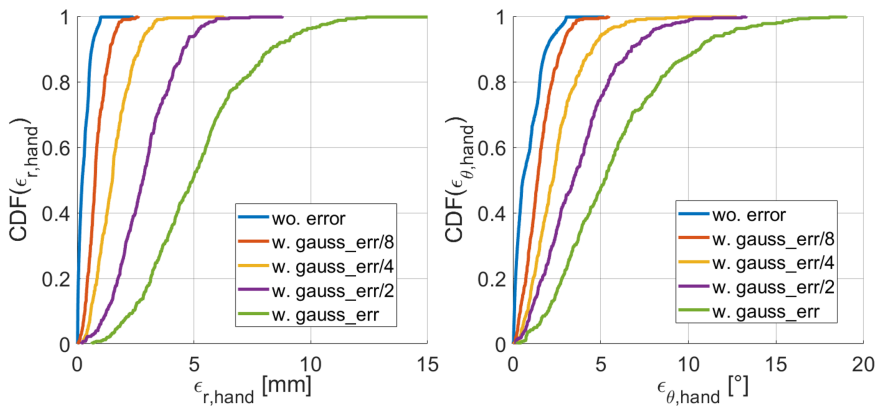

Fig. 14. Euclidean error $\epsilon_{r, \text { hand }}$ on the reconstructed position of the thumb fingertip with respect to hand reference frame, and angular error $\epsilon_{\theta \text {, hand }}$ on the orientation of the thumb distal phalanx. Results are shown for several Gaussian error values, and for the control set without error.
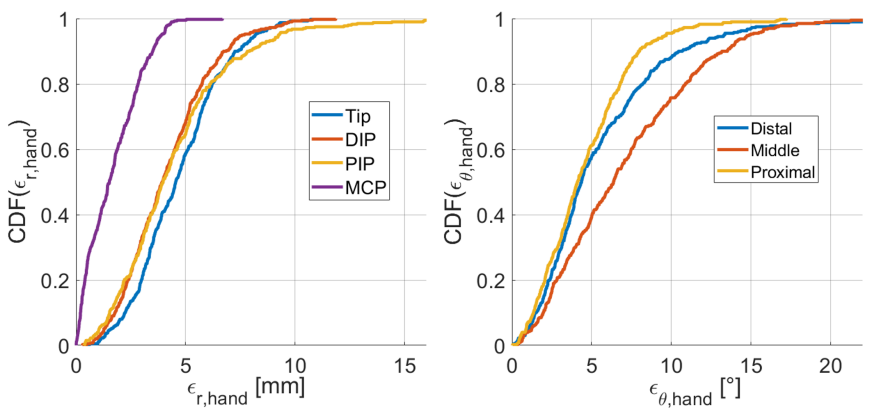

Fig. 15. Euclidean error $\epsilon_{r, \text { hand }}$ on the reconstructed position of joints and tip of the ring finger, with respect to hand reference frame, and angular error $\epsilon_{\theta, \text { hand }}$ on the orientation of the phalanges.
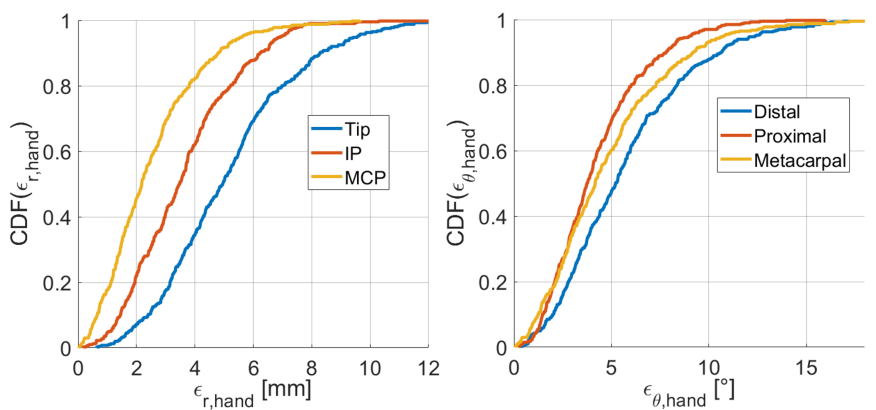

Fig. 16. Euclidean error $\epsilon_{r, \text { hand }}$ on the reconstructed position of joints and tip of the thumb, with respect to hand reference frame, and angular error $\epsilon_{\theta}$, hand on the orientation of the phalanges. 

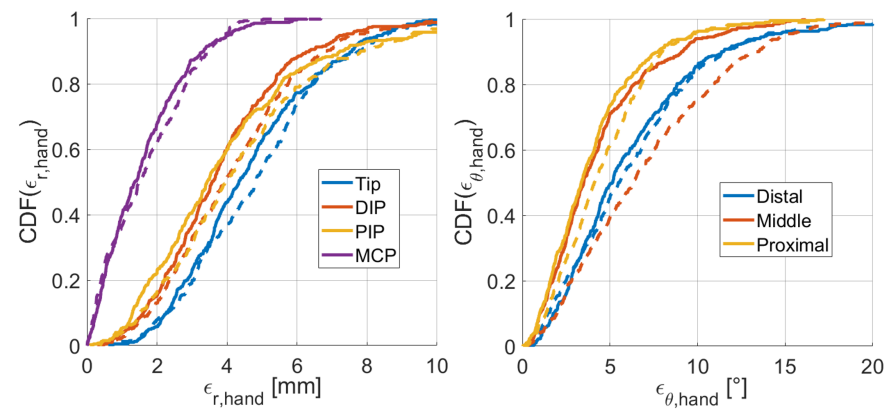

Fig. 17. Euclidean error $\epsilon_{r, \text { hand }}$ on the reconstructed position of joints and tip of the ring finger, with respect to hand reference frame, and angular error $\epsilon_{\theta \text {, hand }}$ on the orientation of the phalanges. Dashed lines: results of inverse kinematics without constraints; solid lines: results obtained by using constraints (14) and (16).

positioning system, and the same inverse kinematics method, the hand pose reconstruction would be more accurate as well.

\section{B. Real hand tracking}

When tracking a real hand, given the limited accuracy of the MPS, in order to obtain a natural hand pose, as explained in Section III-A, we used constraints (14) and (16). We repeated the inverse kinematics on $\boldsymbol{r}_{0, \text { err }}$ and $\hat{\boldsymbol{n}}_{0, \mathrm{err}}$ by using constraints. Results for the Euclidean error on the position of joints, and angular error on the orientation of phalanges are reported in Figure 17, showing only a slight increase of accuracy, except for the orientation of the middle phalanx which is substantially improved. Nevertheless, the resulting pose is globally more natural.

In order to evaluate the accuracy of the hand pose reconstruction, we compared the results of MagIK with those obtained by means of the LeapMotion. The LeapMotion sensor was positioned at the center of the horizontal plane of the MPS. Data from the MPS and the LeapMotion were acquired simultaneously, so as to capture exactly the same gestures of the hand.

Figure 18 shows a visual comparison between the LeapMotion and MagIK hand models. Only for this figure, LeapMotion images have been obtained from the LeapMotion Visualizer utility, while the images of MagIK are from other acquisitions. Here we want just to point out some qualitative aspects. In a), the hand structure is shown for both models; they are quite similar, but the LeapMotion does not include CMC joints for fingers II-V. In b), it is shown the pose reconstruction when the thumb is clenched inside the fist; since the thumb is occluded, the LeapMotion cannot see it, and the resulting reconstruction is wrong. In c), it is shown the pose reconstruction when the thumb and ring fingertips are touching; sometimes, in this or similar poses, the LeapMotion model erroneously flexes also the middle finger; we suppose that the LeapMotion uses a hand model where fingers are not independent; it is impossible for such a situation to occur with MagIK, since each finger is reconstructed independently from others.

A quantitative comparison has been performed on five benchmarks (BM) that we conceived as to represent typical hand gestures. Benchmarks are shown in Figure 19. Each benchmark is defined by a set of characteristic distances. Each

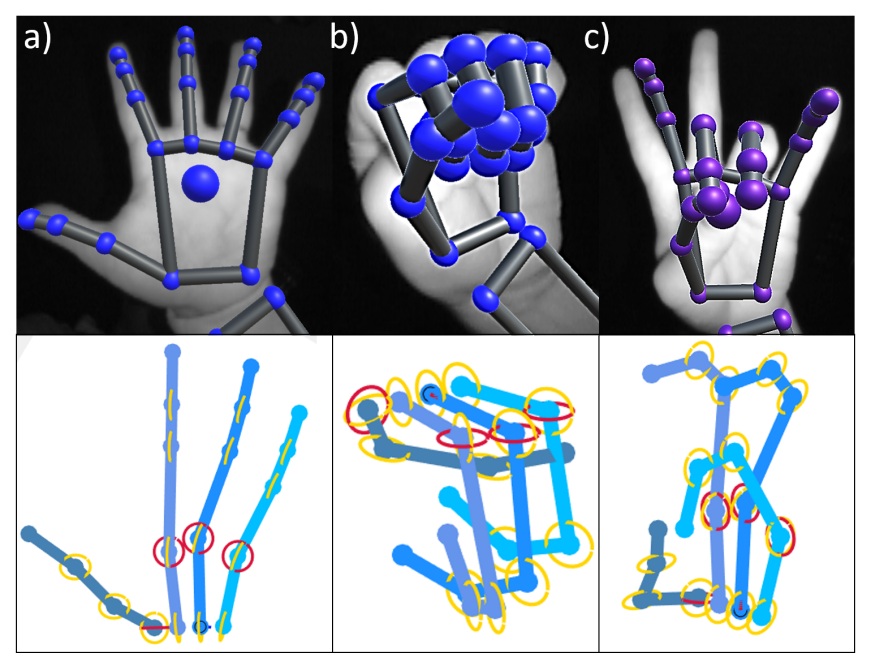

Fig. 18. a) Visual comparison between the hand structure model of the LeapMotion (top) and MagIK (bottom) hand models. b) The LeapMotion does not see the thumb clenched inside the fist. c) Wrong pose reconstruction by the LeapMotion when the thumb and ring fingertips are touching.

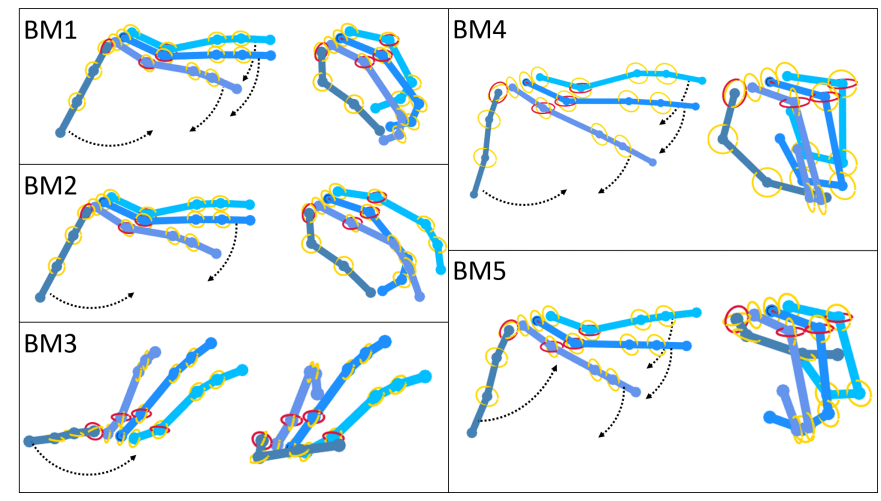

Fig. 19. The five benchmarks that have been conceived to compare the pose reconstruction quality of MagIK with the LeapMotion.

distance is measured in percentage of its maximum, i.e. when all the fingers are fully extended. By using percentages, any possible scale difference between the two systems would be irrelevant for the comparison. Repeated measurements have been performed for each BM.

1) BM1: This benchmark consists in closing and opening all the fingers, keeping the phalanges almost extended, the tip of the thumb touching the other fingertips. We define $d_{1}$ as the average distance between fingertips II-IV; $d_{2}$ as the average distance between the thumb tip and fingertips II-IV. The lesser are $d_{1}$ and $d_{2}$, the better is the performance of the pose recognition system.

MagIK: $d_{1}=54 \% \pm 4 \% ; d_{2}=17 \% \pm 1 \%$.

LeapMotion: $d_{1}=43 \% \pm 2 \% ; d_{2}=17 \% \pm 1 \%$.

The performance of the LeapMotion is partly better, but the gesture is still reconstructed also by MagIK.

2) BM2: Touching the fingertips with the tip of the thumb one at a time. Let $d_{1}$ be the average distance between the thumb tip and fingertips II-IV. The lesser is $d_{1}$, the better is the performance of the system.

MagIK: $d_{1}=14 \% \pm 1 \%$.

LeapMotion: $d_{1}=21 \% \pm 4 \%$. 
MagIK is slightly more accurate than LeapMotion on BM2.

3) BM3: Opposition of the thumb, the most functional gesture of the human hand [51]. Let $d_{1}$ be the average distance between the tip of the thumb and the MCP-IV joint. The lesser is $d_{1}$, the better is the performance of the system.

MagIK: $d_{1}=22 \% \pm 2 \%$.

LeapMotion: $d_{1}=63 \% \pm 9 \%$.

The LeapMotion basically fails at recognizing thumb opposition, while the performance of MagIK is accurate and repeatable.

4) BM4: Clenching the fist by keeping the thumb outside. Let $d_{1}$ be the average distance between tips and MCP joints for fingers II-IV, and $d_{2}$ the average distance between the tip of the thumb and the MCP-III joint. The lesser are both $d_{1}$ and $d_{2}$, the better is the performance, but $d_{2}$ must be greater than $d_{1}$.

MagIK: $d_{1}=39 \% \pm 2 \% ; d_{2}=40 \% \pm 2 \% ; d_{2}-d_{1}=28 \pm 5$ $\mathrm{mm}$.

LeapMotion: $d_{1}=63 \% \pm 1 \% ; d_{2}=76 \% \pm 9 \% ; d_{2}-d_{1}=$ $24 \pm 4 \mathrm{~mm}$.

The difference has been calculated in millimiters, indeed $d_{1}$ and $d_{2}$, measured in percentage, have different normalizations, hence they could not be compared. MagIK is clearly more accurate than the LeapMotion. The fist, as reconstructed by the LeapMotion, is not tightly clenched as it should be.

5) BM5: Clenching the fist by keeping the thumb inside. Let $d_{1}$ be the average distance between tips and MCP joints for fingers II-IV, and $d_{2}$ the average distance between the tip of the thumb and the MCP-IV joint. The lesser are both $d_{1}$ and $d_{2}$, the better is the performance, but $d_{1}$ must be greater than $d_{2}$.

MagIK: $d_{1}=61 \% \pm 3 \% ; d_{2}=16 \% \pm 2 \% ; d_{1}-d_{2}=43 \pm 4$ $\mathrm{mm}$.

LeapMotion: $d_{1}=69 \% \pm 2 \% ; d_{2}=63 \% \pm 11 \% ; d_{1}-d_{2}=$ $-18 \pm 12 \mathrm{~mm}$.

As expected, the LeapMotion system fails to reconstruct the thumb inside the fist, since it is occluded by the other fingers. $d_{1}$ of BM5 is higher than $d_{1}$ of BM4, since the fist in BM5 is not tightly clenched as in BM4, being the thumb kept inside the fist. MagIK is then accurate enough to discriminate between gestures BM4 and BM5.

\section{Conclusions}

A hand tracking method based on a magnetic positioning system has been devised and implemented. The positioning system tracks a set of magnetic nodes, measuring the position (3 DOFs) and the orientation (2 DOFs) of each node. One node for each finger is needed, plus two nodes mounted on the back of the hand, with a total of seven nodes. Our positioning system has six nodes. Hence, we did not track the pinkie, but the technique can be easily extended, since each finger is tracked independently. The system can perform also in non-line-of-sight conditions, as long as no ferromagnetic material altering the field is present within the active volume of the system. A 24 DOFs mechanical model of the hand has been described. Measured positioning data are used to solve the inverse kinematics problem, thus obtaining flexion and abduction angles for each joint. In [53], a hand tracking system is proposed, based on eight sensors equipped with accelerometers, magnetometers and gyroscopes. Sensor data are completed with a set of constraints on joint angles to fully reconstruct the hand poses. In this work we proved that in principle seven 5-DOF sensors are sufficient to reconstruct the hand in all its parts. Constraints are needed only to compensate for the measurement noise of the positioning system. If the positioning accuracy is high enough, constraints are not even needed. We studied the hand tracking accuracy by means of a MC simulation, and then we tested the system by tracking a real hand. The performance of MagIK on gesture recognition has been compared with the results obtained by means of the commercial LeapMotion system. In order to make the comparison, we devised five benchmarks corresponding to characteristic gestures of the human hand. MagIK performed in most of the tests better than the LeapMotion system.

\section{ACKNOWLEDGMENT}

This work was supported by the Italian Ministry of Education, University and Research under Grant PRIN 2015C37B25.

\section{REFERENCES}

[1] W. Chen, C. Yu, C. Tu, Z. Lyu, J. Tang, S. Ou, Y. Fu and Z. Xue, "A Survey on Hand Pose Estimation with Wearable Sensors and ComputerVision-Based Methods," in Sensors vol. 20, no. 4, p. 1074, 2020.

[2] A. Ahmad, C. Migniot and A. Dipanda, "Hand pose estimation and tracking in real and virtual interaction: A review," in Image and Vision Computing, vol. 89, pp. 35-49, July 2019.

[3] M. J. Cheok, Z. Omar and M. H. Jaward, "A review of hand gesture and sign language recognition techniques,", in International Journal of Machine Learning and Cybernetics, vol. 10, pp.131-153, 2019.

[4] A. Ahmad, C. Migniot and A. Dipanda, "Tracking Hands in Interaction with Objects: A Review," in 2017 13th International Conference on Signal-Image Technology \& Internet-Based Systems (SITIS), Jaipur, 2017, pp. 360-369.

[5] T. L. Baldi, S. Scheggi, L. Meli, M. Mohammadi and D. Prattichizzo, "GESTO: A Glove for Enhanced Sensing and Touching Based on Inertial and Magnetic Sensors for Hand Tracking and Cutaneous Feedback," in IEEE Transactions on Human-Machine Systems, vol. 47, no. 6, pp. 1066 1076, Dec. 2017.

[6] T. Piumsomboon, A. Clark, M. Billinghurst and A. Cockburn, "UserDefined Gestures for Augmented Reality,“ in P. Kotzé, G. Marsden, G. Lindgaard, J. Wesson and M. Winckler (eds), "Human-Computer Interaction - INTERACT 2013", INTERACT 2013. Lecture Notes in Computer Science, vol. 8118, Springer, Berlin, Heidelberg.

[7] C. Wu, C. Hsu, T. Lee et al., "A virtual reality keyboard with realistic haptic feedback in a fully immersive virtual environment," in Virtual Reality, vol. 21, pp. 19-29, 2017.

[8] S. Lee, K. Park, J. Lee and K. Kim, "User Study of VR Basic Controller and Data Glove as Hand Gesture Inputs in VR Games," in 2017 International Symposium on Ubiquitous Virtual Reality (ISUVR), Nara, 2017, pp. 1-3.

[9] I. Yavrucuk, E. Kubali and O. Tarimci, "A low cost flight simulator using virtual reality tools," in IEEE Aerospace and Electronic Systems Magazine, vol. 26, no. 4, pp. 10-14, April 2011.

[10] H. Rijpkema and M. Girard, "Computer animation of knowledgebased human grasping," in Proceedings of the 18th annual conference on Computer graphics and interactive techniques (SIGGRAPH '91). Association for Computing Machinery, New York, NY, USA, 339-348, 1991.

[11] J. Lee and T. L. Kunii, "Constraint-Based Hand Animation," in N. M. Thalmann and D. Thalmann (eds), "Models and Techniques in Computer Animation," Springer Japan, Tokyo, 1993, pp. 110-127.

[12] J. Kim and J. Park, "Direct and realistic handover of a virtual object," in 2016 IEEE/RSJ International Conference on Intelligent Robots and Systems (IROS), Daejeon, 2016, pp. 994-999. 
[13] P. Bellitti et al., "A Wearable and Wirelessly Powered System for Multiple Finger Tracking," in IEEE Transactions on Instrumentation and Measurement, vol. 69, no. 5, pp. 2542-2551, May 2020.

[14] W. Wang, R. Li, Z. M. Diekel, Y. Chen, Z. Zhang and Y. Jia, "Controlling Object Hand-Over in Human-Robot Collaboration Via Natural Wearable Sensing," in IEEE Transactions on Human-Machine Systems, vol. 49, no. 1, pp. 59-71, Feb. 2019.

[15] P. M. Kebria, H. Abdi, M. M. Dalvand, A. Khosravi and S. Nahavandi, "Control Methods for Internet-Based Teleoperation Systems: A Review," in IEEE Transactions on Human-Machine Systems, vol. 49, no. 1, pp. 32-46, Feb. 2019.

[16] R. B. Warrier and S. Devasia, "Iterative Learning From Novice Human Demonstrations for Output Tracking," in IEEE Transactions on HumanMachine Systems, vol. 46, no. 4, pp. 510-521, Aug. 2016.

[17] H. G. Kortier, V. I. Sluiter, D. Roetenberg, et al., "Assessment of hand kinematics using inertial and magnetic sensors," in Journal of NeuroEngineering and Rehabilitation 11, 70, 2014.

[18] Yu Su et al., "3-D motion system ("data-gloves"): application for Parkinson's disease," in IEEE Transactions on Instrumentation and Measurement, vol. 52, no. 3, pp. 662-674, June 2003.

[19] J. Li et al., "Three-Dimensional Pattern Features in Finger Tapping Test for Patients with Parkinson's disease," in 2020 42nd Annual International Conference of the IEEE Engineering in Medicine \& Biology Society $(E M B C)$, Montreal, QC, Canada, 2020, pp. 3676-3679.

[20] A. Moshkova, A. Samorodov, N. Voinova, A. Volkov, E. Ivanova and E. Fedotova, "Parkinson's Disease Detection by Using Machine Learning Algorithms and Hand Movement Signal from LeapMotion Sensor," in 2020 26th Conference of Open Innovations Association (FRUCT), Yaroslavl, Russia, 2020, pp. 321-327.

[21] J. Zhou, F. Malric and S. Shirmohammadi, "A New Hand-Measurement Method to Simplify Calibration in CyberGlove-Based Virtual Rehabilitation," in IEEE Transactions on Instrumentation and Measurement, vol. 59, no. 10, pp. 2496-2504, Oct. 2010.

[22] Y. Rybarczyk, J. L. Pérez Medina, L. Leconte, K. Jimenes, M. González and D. Esparza, "Implementation and Assessment of an Intelligent Motor Tele-Rehabilitation Platform," in Electronics 2019, 8, 58.

[23] M. A. Ahmed, B. B. Zaidan, A. A. Zaidan, M. M. Salih and M. M. Lakulu, "A Review on Systems-Based Sensory Gloves for Sign Language Recognition State of the Art between 2007 and 2017," in Sensors 2018, 18, 2208.

[24] Z. Lu, X. Chen, Q. Li, X. Zhang and P. Zhou, "A Hand Gesture Recognition Framework and Wearable Gesture-Based Interaction Prototype for Mobile Devices," in IEEE Transactions on Human-Machine Systems, vol. 44, no. 2, pp. 293-299, April 2014.

[25] S. S. Rautaray and A. Agrawal, "Vision based hand gesture recognition for human computer interaction: a survey," in Artificial Intelligence Review, vol. 43, pp. 1-54, 2015.

[26] N. H. Dardas and N. D. Georganas, "Real-Time Hand Gesture Detection and Recognition Using Bag-of-Features and Support Vector Machine Techniques," in IEEE Transactions on Instrumentation and Measurement, vol. 60, no. 11, pp. 3592-3607, Nov. 2011.

[27] L. Bretzner, I. Laptev and T. Lindeberg, "Hand gesture recognition using multi-scale colour features, hierarchical models and particle filtering," in Proceedings of Fifth IEEE International Conference on Automatic Face Gesture Recognition, Washington, DC, USA, 2002.

[28] www.ultraleap.com/product/leap-motion-controller/

[29] P. P. Valentini and E. Pezzuti, "Accuracy in fingertip tracking using Leap Motion Controller for interactive virtual applications," in Int. J. Interact. Des. Manuf., 11, 641-650, 2017.

[30] M. Borghetti, E. Sardini and M. Serpelloni, "Sensorized Glove for Measuring Hand Finger Flexion for Rehabilitation Purposes," in IEEE Transactions on Instrumentation and Measurement, vol. 62, no. 12, pp. 3308-3314, Dec. 2013.

[31] L. Sbernini, A. Pallotti and G. Saggio, "Evaluation of a Stretch Sensor for its inedited application in tracking hand finger movements," in 2016 IEEE International Symposium on Medical Measurements and Applications (MeMeA), Benevento, 2016.

[32] B.-S. Lin, I.-J. Lee, S.-Y. Yang, Y.-C. Lo, J. Lee and J.-L. Chen, "Design of an Inertial-Sensor-Based Data Glove for Hand Function Evaluation," in Sensors, 2018, 18, 1545.

[33] P. Bellitti, M. Bona, M. Borghetti, E. Sardini and M. Serpelloni, "Analysis for a Modular Wearable Finger 3D Motion Tracking System," in Proceedings 2018, 2, 1051.

[34] Z. Xiao, W. Hu, C. Liu, H. Yu and C. Li, "Noncontact Human-Machine Interface With Planar Probing Coils in a Differential Sensing Architecture," in IEEE Transactions on Instrumentation and Measurement, vol. 67, no. 4, pp. 956-964, April 2018.
[35] M. A. Simão, P. Neto and O. Gibaru, "Unsupervised Gesture Segmentation by Motion Detection of a Real-Time Data Stream," in IEEE Transactions on Industrial Informatics, vol. 13, no. 2, pp. 473-481, April 2017.

[36] M. Hassan, K. Assaleh and T. Shanableh, "Multiple Proposals for Continuous Arabic Sign Language Recognition," in Sensing and Imaging, 20, 4, 2019.

[37] C-S. Fahn and H. Sun, "Development of a data glove with reducing sensors based on magnetic induction," in IEEE Transactions on Industrial Electronics, vol. 52, no. 2, pp. 585-594, April 2005.

[38] C-S. Fahn and H. Sun, "Development of a Fingertip Glove Equipped with Magnetic Tracking Sensors," in Sensors 2010, 10(2), 1119-1140.

[39] F. Santoni, A. De Angelis, A. Moschitta and P. Carbone, "A MultiNode Magnetic Positioning System with a Distributed Data Acquisition Architecture," in Sensors, 2020, 20, 6210

[40] G. De Angelis, A. De Angelis, A. Moschitta and P. Carbone, "Comparison of Measurement Models for 3D Magnetic Localization and Tracking.", in Sensors 2017, 17(11), 2527

[41] F. Santoni, A. De Angelis, I. Skog, A. Moschitta and P. Carbone, "Calibration and Characterization of a Magnetic Positioning System Using a Robotic Arm," in IEEE Transactions on Instrumentation and Measurement, vol. 68, no. 5, pp. 1494-1502, May 2019.

[42] L. Ferrigno et al., "A real-time tracking system for tremor and trajectory estimation in Parkinson's disease affected patients", in 2020 IEEE International Symposium on Medical Measurements and Applications (MeMeA), Bari, Italy, 2020, pp. 1-6.

[43] G. De Angelis, A. De Angelis, A. Comuniello, A. Moschitta and P. Carbone, "Simultaneous RSS Measurements Using Multiple Inductively Coupled Coils," in 2019 IEEE International Symposium on Measurements \& Networking $(M \& N)$, Catania, Italy, 2019, pp. 1-6.

[44] D. Capriglione, G. Cerro, L. Ferrigno, F. Milano and A. Moschitta, "A multi-frequency approach to mitigate the performance degradation of a magnetic positioning system under $\mathrm{CW}$ disturbance conditions," Measurements, vol. 161, 2020, 107842.

[45] Y. Yasumuro, Q. Chen and K. Chihara, "3D modeling of human hand with motion constraints," in Proceedings. International Conference on Recent Advances in 3-D Digital Imaging and Modeling (Cat. No.97TB100134), Ottawa, Ontario, Canada, 1997, pp. 275-282.

[46] P. K. Parida and B. B. Biswal, "Design and Analysis of a Multifingered Robot Hand", in International Journal of Robotics and Automation, vol. 1, no. 2, June 2012, pp.69-77.

[47] F. C. Chen, S. Appendino, A. Battezzato, A. Favetto, M. Mousavi and F. Pescarmona, "Constraint Study for a Hand Exoskeleton: Human Hand Kinematics and Dynamics," in Journal of Robotics, vol. 2013, Article ID 910961, 17 pages, 2013.

[48] S. Cobos, M. Ferre, M. A. Sanchez Uran, J. Ortego and C. Pena, "Efficient human hand kinematics for manipulation tasks," in 2008 IEEE/RSJ International Conference on Intelligent Robots and Systems, Nice, 2008, pp. 2246-2251.

[49] J. Zhou, J. Yi, X. Chen, Z. Liu and Z. Wang, "BCL-13: A 13-DOF Soft Robotic Hand for Dexterous Grasping and In-Hand Manipulation," in IEEE Robotics and Automation Letters, vol. 3, no. 4, pp. 3379-3386, Oct. 2018.

[50] J. J. Craig, "Introduction to Robotics, Mechanics and Control," Pearson Education International, 3rd edition, 1986.

[51] A. I. Kapandji, "The Physiology of the Joints," Church Livingstone Elsevier, 6th edition, 2007

[52] J. Lee and T. L. Kunii, "Model-based analysis of hand posture," in IEEE Computer Graphics and Applications, vol. 15, no. 5, pp. 77-86, Sept. 1995.

[53] C-E. Hrabia, K. Wolf and M. Wilhelm, "Whole hand modeling using 8 wearable sensors: biomechanics for hand pose prediction," in Proceedings of the 4th Augmented Human International Conference (AH '13), Association for Computing Machinery, New York, NY, USA, 21-28, 2013.

[54] T. M. Greiner, "Hand Anthropometry of U.S. Army Personnel," Technical Report Natick/TR-92/011, U.S. Army Natick Research, Development and Engineering Center Natick, Massachussetts, December 1991. 